\title{
Constrained minimum Riesz and Green energy problems for vector measures associated with a generalized condenser
}

\author{
Bent Fuglede and Natalia ZorII \\ (Received December 28, 2017) \\ (Revised May 25, 2019)
}

\begin{abstract}
For a finite collection $\mathbf{A}=\left(A_{i}\right)_{i \in I}$ of locally closed sets in $\mathbb{R}^{n}, n \geqslant 3$, with the sign $s_{i}= \pm 1$ prescribed such that the oppositely charged plates are mutually disjoint, we consider the minimum energy problem relative to the $\alpha$-Riesz kernel $|x-y|^{\alpha-n}$, $\alpha \in(0,2]$, over positive vector Radon measures $\boldsymbol{\mu}=\left(\mu^{i}\right)_{i \in I}$ such that each $\mu^{i}, i \in I$, is carried by $A_{i}$ and normalized by $\mu^{i}\left(A_{i}\right)=a_{i} \in(0, \infty)$, while the interaction between $\mu^{i}, i \in I$, is determined by the matrix $\left(s_{i} s_{j}\right)_{i, j \in I}$. We show that, though the closures of oppositely charged plates may intersect each other even in a set of nonzero capacity, this problem has a solution $\lambda_{\mathbf{A}}^{\xi}=\left(\lambda_{\mathbf{A}}^{i}\right)_{i \in I}$ (also in the presence of an external field) if we restrict ourselves to $\boldsymbol{\mu}$ with $\mu^{i} \leqslant \xi^{i}, i \in I$, where the constraint $\xi=\left(\xi^{i}\right)_{i \in I}$ is properly chosen. We establish the sharpness of the sufficient conditions on the solvability thus obtained, provide descriptions of the weighted vector $\alpha$-Riesz potentials of the solutions, single out their characteristic properties, and analyze the supports of the $\lambda_{\mathbf{A}}^{i}, i \in I$. Our approach is based on the simultaneous use of the vague topology and an appropriate semimetric structure defined in terms of the $\alpha$-Riesz energy on a set of vector measures associated with $\mathbf{A}$, as well as on the establishment of an intimate relationship between the constrained minimum $\alpha$-Riesz energy problem and a constrained minimum $\alpha$-Green energy problem, suitably formulated. The results are illustrated by examples.
\end{abstract}

\section{Introduction}

The purpose of this paper is to study minimum energy problems with external fields (also known as weighted minimum energy problems or as the Gauss variational problems) relative to the $\alpha$-Riesz kernel $\kappa_{\alpha}(x, y):=|x-y|^{\alpha-n}$ of order $\alpha \in(0,2]$ on $\mathbb{R}^{n}, n \geqslant 3$, where $|x-y|$ denotes the Euclidean distance between $x, y \in \mathbb{R}^{n}$ and the infimum is taken over classes of vector measures $\boldsymbol{\mu}=\left(\mu^{i}\right)_{i \in I}$ associated with a generalized condenser $\mathbf{A}=\left(A_{i}\right)_{i \in I}$ in $\mathbb{R}^{n}$ and normalized by $\mu^{i}\left(A_{i}\right)=a_{i} \in(0, \infty), i \in I$. More precisely, an ordered finite collection A of locally closed sets $A_{i} \subset \mathbb{R}^{n}, i \in I$, termed plates, with the sign $s_{i}= \pm 1$ prescribed is said to be a generalized condenser if the oppositely signed

2010 Mathematics Subject Classification. 31C15.

Key words and phrases. Constrained minimum energy problems, condensers with touching plates, vector measures, external fields, perfect kernels, $\alpha$-Riesz kernels, $\alpha$-Green kernels. 
plates are mutually disjoint, while a vector measure $\boldsymbol{\mu}=\left(\mu^{i}\right)_{i \in I}$ is said to be associated with $\mathbf{A}$ if each $\mu^{i}, i \in I$, is a positive scalar Radon measure (charge) on $\mathbb{R}^{n}$, carried by $A_{i}$. In accordance with an electrostatic interpretation of a condenser, we say that the interaction between the components $\mu^{i}, i \in I$, of such $\boldsymbol{\mu}$ is determined by the matrix $\left(s_{i} s_{j}\right)_{i, j \in I}$, so that the f-weighted $\alpha$-Riesz energy of $\boldsymbol{\mu}$ is defined by

$$
G_{\kappa_{\alpha}, \mathbf{f}}(\boldsymbol{\mu}):=\sum_{i, j \in I} s_{i} s_{j} \int|x-y|^{\alpha-n} d\left(\mu^{i} \otimes \mu^{j}\right)(x, y)+2 \sum_{i \in I} \int f_{i} d \mu^{i},
$$

where $\mathbf{f}=\left(f_{i}\right)_{i \in I}$, each $f_{i}: \mathbb{R}^{n} \rightarrow[-\infty, \infty]$ being a universally measurable function treated as an external field acting on the charges carried by the $A_{i}$.

The difficulties appearing in the course of our investigation are caused by the fact that a short-circuit may occur between $A_{i}$ and $A_{j}$ with $s_{i} s_{j}=-1$, because these conductors may have zero Euclidean distance. See Theorem 5.1 below providing an example of a generalized condenser with no $\alpha$-Riesz energy minimizer. It is therefore meaningful to ask what kinds of additional requirements on the objects in question will prevent this blow-up effect, and secure that a solution to the corresponding $\mathbf{f}$-weighted minimum $\alpha$-Riesz energy problem does exist.

We show that, though the closures of oppositely charged plates may intersect each other even in a set of nonzero $\alpha$-Riesz capacity, such minimum energy problem is nevertheless solvable (no short-circuit occurs) if we restrict ourselves to $\boldsymbol{\mu}$ with $\mu^{i} \leqslant \xi^{i}, i \in I$, where the constraint $\xi=\left(\xi^{i}\right)_{i \in I}$ is properly chosen (see Sections 3.5 and 5.2 for a formulation of the constrained problem). Sufficient conditions for the existence of solutions $\lambda_{\mathbf{A}}^{\xi}=\left(\lambda_{\mathbf{A}}^{i}\right)_{i \in I}$ to the constrained minimum $\alpha$-Riesz energy problem are established in Theorems 7.1 and 7.6; those conditions are shown in Theorem 7.2 to be sharp. The uniqueness of solutions is studied in Lemma 3.6 and Corollary 3.1. We also provide descriptions of the $\mathbf{f}$-weighted vector $\alpha$-Riesz potentials of the solutions $\lambda_{\mathbf{A}}^{\xi}$, single out their characteristic properties, and analyze the supports of the components $\lambda_{\mathrm{A}}^{i}, i \in I$ (Theorems 7.3, 7.4 and 7.5). The results are illustrated in Examples 10.1 and 10.2 .

In particular, let $\mathbf{A}=\left(A_{1}, A_{2}\right)$ be a generalized condenser with the positive plate $A_{1}:=D$ and the negative plate $A_{2}:=\mathbb{R}^{n} \backslash D, D$ being an (open connected) bounded domain in $\mathbb{R}^{n}$ with $m_{n}(D)>1, m_{n}$ being the Lebesgue measure on $\mathbb{R}^{n}$, and let $\mathbf{f}=\mathbf{0}$. Then inf $G_{\kappa_{\alpha}, \mathbf{f}}(\boldsymbol{\mu})$ over all $\boldsymbol{\mu}=\left(\mu^{1}, \mu^{2}\right)$ associated with A and normalized by $\mu^{i}\left(A_{i}\right)=1, i=1,2$, is an actual minimum (although $\left.A_{2} \cap \mathrm{Cl}_{\mathbb{R}^{n}} A_{1}=\partial D\right)$ if we require additionally that $\mu^{1} \leqslant \xi^{1}:=\left.m_{n}\right|_{D}$ and $\mu^{2} \leqslant \xi^{2}$, where $\xi^{2}$ is a positive Radon measure carried by $A_{2}$ and possessing the property $\xi^{2} \geqslant\left(\left.m_{n}\right|_{D}\right)^{A_{2}}$ (cf. Theorems 7.1 and 7.6). Here $\left.m_{n}\right|_{D}$ is the 
restriction of $m_{n}$ on $D$, and $\left(\left.m_{n}\right|_{D}\right)^{A_{2}}$ the $\alpha$-Riesz balayage of $\left.m_{n}\right|_{D}$ onto $A_{2}$. Throughout the paper $\partial Q$ denotes the boundary of a set $Q \subset \mathbb{R}^{n}$ in the topology of $\mathbb{R}^{n}$.

The approach developed below is mainly based on the simultaneous use of the vague topology and an appropriate (semi)metric structure defined in terms of the $\alpha$-Riesz energy on a set of vector measures associated with a generalized condenser (see Section 3.2 for a definition of such a (semi)metric structure ${ }^{1}$ ), as well as on the establishment of an intimate relationship between the constrained minimum $\alpha$-Riesz energy problem and a constrained minimum $\alpha$-Green energy problem, suitably formulated. Regarding the latter problem, crucial to the arguments applied in its investigation is the perfectness of the $\alpha$-Green kernel $g_{D}^{\alpha}$ on an arbitrary open set $D$, established recently by the present authors [15], which amounts to the completeness in the topology determined by the energy norm $\|v\|_{g_{D}^{\alpha}}:=\sqrt{g_{D}^{\alpha}(v, v)}$ of the cone of all positive scalar Radon measures $v$ on $D$ with finite $\alpha$-Green energy $g_{D}^{\alpha}(v, v):=\int g_{D}^{\alpha}(x, y) d(v \otimes v)(x, y)<\infty$.

\section{Preliminaries}

Let $X$ be a locally compact (Hausdorff) space [2, Chapter I, Section 9, $\mathrm{n}^{\circ}$ 7], to be specified below, and $\mathfrak{M}(X)$ the linear space of all real-valued scalar (signed) Radon measures $\mu$ on $X$, equipped with the vague topology, i.e. the topology of pointwise convergence on the class $C_{0}(X)$ of all continuous functions $^{2}$ on $X$ with compact support. We refer to $[3,10]$ for the theory of measures and integration on $X$, to be used throughout the paper (see also [11] for a short survey).

For the purposes of the present study it is enough to assume that $X$ is metrizable and countable at infinity, where the latter means that $X$ can be represented as a countable union of compact sets [2, Chapter I, Section 9, $\mathrm{n}^{\circ}$ 9]. Then the vague topology on $\mathfrak{M}(X)$ satisfies the first axiom of countability [14, Remark 2.4], and the vague convergence is entirely determined by convergence of sequences. The vague topology on $\mathfrak{M}(X)$ is Hausdorff; hence, a vague limit of any sequence in $\mathfrak{M}(X)$ is unique (whenever it exists).

Let $\mu^{+}$and $\mu^{-}$denote the positive and negative parts of a measure $\mu \in \mathfrak{M}(X)$ in the Hahn-Jordan decomposition, $|\mu|:=\mu^{+}+\mu^{-}$its total varia-

\footnotetext{
${ }^{1}$ A key observation behind that definition is the fact that there corresponds to every positive vector measure $\boldsymbol{\mu}=\left(\mu^{i}\right)_{i \in I}$ of finite energy associated with A a scalar (signed) Radon measure $R \boldsymbol{\mu}:=\sum_{i \in I} s_{i} \mu^{i}$ on $\mathbb{R}^{n}$, and the mapping $R: \boldsymbol{\mu} \mapsto R \boldsymbol{\mu}$ preserves the corresponding energy semimetric (see Theorem 3.1). This approach extends that from [22]-[26] where the closures of the oppositely charged plates were assumed to be mutually disjoint.

${ }^{2}$ When speaking of a continuous numerical function we understand that the values are finite real numbers.
} 
tion, and $S(\mu)=S_{X}^{\mu}$ its support. A measure $\mu$ is said to be bounded if $|\mu|(X)<\infty$.

Let $\mathfrak{M}^{+}(X)$ stand for the (convex, vaguely closed) cone of all positive $\mu \in \mathfrak{M}(X)$, and let $\Psi(X)$ consist of all lower semicontinuous (1.s.c.) functions $\psi: X \rightarrow(-\infty, \infty]$, nonnegative unless $X$ is compact.

Lemma 2.1 (see e.g. [11, Section 1.1]). For any $\psi \in \Psi(X)$ the mapping $\mu \mapsto\langle\psi, \mu\rangle:=\int \psi d \mu$ is vaguely l.s.c. on $\mathfrak{M}^{+}(X)$.

We define a (function) kernel on $X$ as a symmetric positive function $\kappa$ from $\Psi(X \times X)$. Given $\mu, v \in \mathfrak{M}(X)$, we denote by $\kappa(\mu, v)$ and $\kappa(\cdot, \mu)$ the mutual energy and the potential relative to the kernel $\kappa$, respectively, i.e. ${ }^{3}$

$$
\begin{aligned}
& \kappa(\mu, v):=\int \kappa(x, y) d(\mu \otimes v)(x, y), \\
& \kappa(x, \mu):=\int \kappa(x, y) d \mu(y), \quad x \in X .
\end{aligned}
$$

Note that $\kappa(x, \mu)$ is well defined provided that $\kappa\left(x, \mu^{+}\right)$or $\kappa\left(x, \mu^{-}\right)$is finite, and then $\kappa(x, \mu)=\kappa\left(x, \mu^{+}\right)-\kappa\left(x, \mu^{-}\right)$. In particular, if $\mu \in \mathfrak{M}^{+}(X)$, then $\kappa(x, \mu)$ is defined everywhere and represents a 1.s.c. positive function on $X$ (see Lemma 2.1). Also observe that $\kappa(\mu, v)$ is well defined and equal to $\kappa(v, \mu)$ provided that $\kappa\left(\mu^{+}, v^{+}\right)+\kappa\left(\mu^{-}, v^{-}\right)$or $\kappa\left(\mu^{+}, v^{-}\right)+\kappa\left(\mu^{-}, v^{+}\right)$is finite. For $\mu=v, \kappa(\mu, v)$ becomes the energy $\kappa(\mu, \mu)$ of $\mu$. Let $\mathscr{E}_{K}(X)$ consist of all $\mu \in \mathfrak{M}(X)$ whose energy $\kappa(\mu, \mu)$ is finite, which by definition means that the kernel $\kappa$ is $(|\mu| \otimes|\mu|)$-integrable, i.e. $\kappa(|\mu|,|\mu|)<\infty$, and let $\mathscr{E}_{\kappa}^{+}(X):=$ $\mathscr{E}_{K}(X) \cap \mathfrak{M}^{+}(X)$.

Given a set $Q \subset X$, let $\mathfrak{M}^{+}(Q ; X)$ consist of all $\mu \in \mathfrak{M}^{+}(X)$ carried by $Q$, which means that $X \backslash Q$ is locally $\mu$-negligible, or equivalently that $Q$ is $\mu$-measurable and $\mu=\left.\mu\right|_{Q}$, where $\left.\mu\right|_{Q}=1_{Q} \cdot \mu$ is the restriction of $\mu$ on $Q$ [3, Chapter V, Section 5, $\mathrm{n}^{\circ} 3$, Example]. (Here $1_{Q}$ denotes the indicator function of $Q$.) If $Q$ is closed, then $\mu$ is carried by $Q$ if and only if it is supported by $Q$, i.e. $S(\mu) \subset Q$. It follows from the countability of $X$ at infinity that the concept of local $\mu$-negligibility coincides with that of $\mu$-negligibility; and hence $\mu \in \mathfrak{M}^{+}(Q ; X)$ if and only if $\mu^{*}(X \backslash Q)=0, \mu^{*}(\cdot)$ being the outer measure of a set. Denoting by $\mu_{*}(\cdot)$ the inner measure of a set, for any $\mu \in \mathfrak{M i}^{+}(Q ; X)$ we thus get

$$
\mu^{*}(Q)=\mu_{*}(Q)=: \mu(Q)
$$

\footnotetext{
${ }^{3}$ When introducing notation about numerical quantities we assume the corresponding object on the right to be well defined (as a finite real number or $\pm \infty$ ).
} 
Write $\mathscr{E}_{\kappa}^{+}(Q ; X):=\mathscr{E}_{\kappa}(X) \cap \mathfrak{M}^{+}(Q ; X), \quad \mathfrak{M}^{+}(Q, q ; X):=\left\{\mu \in \mathfrak{M}^{+}(Q ; X): \mu(Q)\right.$ $=q\}$ and $\mathscr{E}_{\kappa}^{+}(Q, q ; X):=\mathscr{E}_{\kappa}(X) \cap \mathfrak{M}^{+}(Q, q ; X)$, where $q \in(0, \infty)$.

Among the variety of potential-theoretic principles investigated for example in the comprehensive work by Ohtsuka [21] (see also the references therein), in the present study we shall only need the following two:

- A kernel $\kappa$ is said to satisfy the complete maximum principle (introduced by Cartan and Deny [6]) if for any $\mu \in \mathscr{E}_{\kappa}^{+}(X)$ and $v \in \mathfrak{M i}^{+}(X)$ such that $\kappa(x, \mu) \leqslant \kappa(x, v)+c \mu$-a.e., where $c \geqslant 0$ is a constant, the same inequality holds everywhere on $X$.

- A kernel $\kappa$ is said to be positive definite if $\kappa(\mu, \mu) \geqslant 0$ for every (signed) measure $\mu \in \mathfrak{M}(X)$ for which the energy is well defined; and $\kappa$ is said to be strictly positive definite, or to satisfy the energy principle if in addition $\kappa(\mu, \mu)>0$ except for $\mu=0$.

Unless explicitly stated otherwise, in all that follows we assume a kernel $\kappa$ to satisfy the energy principle. Then $\mathscr{E}_{K}(X)$ forms a pre-Hilbert space with the inner product $\kappa(\mu, v)$ and the energy norm $\|\mu\|_{\kappa}:=\sqrt{\kappa(\mu, \mu)}$ (see [11]). The (Hausdorff) topology on $\mathscr{E}_{\kappa}(X)$ determined by the norm $\|\cdot\|_{\kappa}$ is termed strong.

In contrast to $[12,13]$ where capacity has been treated as a functional acting on positive numerical functions on $X$, in the present study we use the (standard) concept of capacity as a set function. Thus the (inner) capacity of a set $Q \subset X$ relative to the kernel $\kappa$, denoted $c_{\kappa}(Q)$, is defined by

$$
c_{\kappa}(Q):=\left[\inf _{\mu \in \mathscr{E}_{\kappa}^{+}(Q, 1 ; X)} \kappa(\mu, \mu)\right]^{-1}
$$

(see e.g. $[11,21]$ ). Then $0 \leqslant c_{\kappa}(Q) \leqslant \infty$. (As usual, here and in the sequel the infimum over the empty set is taken to be $+\infty$. We also set $1 /(+\infty)=0$ and $1 / 0=+\infty$.) Because of the strict positive definiteness of the kernel $\kappa$,

$$
c_{\kappa}(K)<\infty \quad \text { for every compact } K \subset X .
$$

Furthermore, by [11, p. 153, Eq. 2],

$$
c_{\kappa}(Q)=\sup c_{\kappa}(K) \quad(K \subset Q, K \text { compact }) .
$$

We shall often use the fact that $c_{\kappa}(Q)=0$ if and only if $\mu_{*}(Q)=0$ for every $\mu \in \mathscr{E}_{\kappa}^{+}(X)$, see [11, Lemma 2.3.1].

As in [19, p. 134], we call a measure $\mu \in \mathfrak{M}(X) c_{\kappa}$-absolutely continuous if $\mu(K)=0$ for every compact set $K \subset X$ with $c_{K}(K)=0$. It follows from (2.3) that for such $\mu,|\mu|_{*}(Q)=0$ for every $Q \subset X$ with $c_{\kappa}(Q)=0$. Hence, every $\mu \in \mathscr{E}_{\kappa}(X)$ is $c_{\kappa}$-absolutely continuous; but not conversely [19, pp. 134135]. 
Definition 2.1. Following [11], we call a (strictly positive definite) kernel $\kappa$ perfect if every strong Cauchy sequence in $\mathscr{E}_{\kappa}^{+}(X)$ converges strongly to any of its vague cluster points ${ }^{4}$.

REMARK 2.1. On $X=\mathbb{R}^{n}, n \geqslant 3$, the $\alpha$-Riesz kernel $\kappa_{\alpha}(x, y)=|x-y|^{\alpha-n}$, $\alpha \in(0, n)$, is strictly positive definite and moreover perfect $[7,8]$; thus so is the Newtonian kernel $\kappa_{2}(x, y)=|x-y|^{2-n}[5]$. Recently it has been shown by the present authors that if $X=D$ where $D$ is an open set in $\mathbb{R}^{n}, n \geqslant 3$, and $g_{D}^{\alpha}$, $\alpha \in(0,2]$, is the $\alpha$-Green kernel on $D\left[19\right.$, Chapter IV, Section 5], then $\kappa=g_{D}^{\alpha}$ likewise is strictly positive definite and moreover perfect [15, Theorems 4.9, 4.11]. See also [14, Remark 2.2] for some other examples of perfect kernels.

THEOREM 2.1 (see [11]). If a kernel $\kappa$ on a locally compact space $X$ is perfect, then the cone $\mathscr{E}_{\kappa}^{+}(X)$ is strongly complete and the strong topology on $\mathscr{E}_{\kappa}^{+}(X)$ is finer than the (induced) vague topology on $\mathscr{E}_{\kappa}^{+}(X)$.

REMARK 2.2. In contrast to Theorem 2.1, for a perfect kernel $\kappa$ the whole pre-Hilbert space $\mathscr{E}_{K}(X)$ is in general strongly incomplete, and this is the case even for the $\alpha$-Riesz kernel of order $\alpha \in(1, n)$ on $\mathbb{R}^{n}, n \geqslant 3$ (see [5] and [19, Theorem 1.19]). When speaking of a completion of $\mathscr{E}_{K_{\alpha}}\left(\mathbb{R}^{n}\right)$, one needs to consider e.g. tempered distributions of finite Deny-Schwartz energy defined with the aid of the Fourier transform [7]. Recently it has also been shown that if we restrict ourselves to $v \in \mathscr{E}_{\kappa_{\alpha}}\left(\mathbb{R}^{n}\right)$ such that $S_{\mathbb{R}^{n}}^{v} \subset D, D$ being a bounded domain in $\mathbb{R}^{n}$, then the pre-Hilbert space of all those $v$ can be isometrically imbedded into its completion, the Sobolev space $\tilde{H}^{-\alpha / 2}(D)$, see [18, Corollary 3.3].

REMARK 2.3. The concept of perfect kernel is an efficient tool in minimum energy problems over classes of positive scalar Radon measures with finite energy. Indeed, if $Q \subset X$ is closed, $c_{\kappa}(Q) \in(0, \infty)$, and $\kappa$ is perfect, then the minimum energy problem $(2.1)$ has a unique solution $\lambda_{Q}$, termed the (inner) $\kappa$-capacitary measure on $Q$ [11, Theorem 4.1]. Later the concept of perfectness has been shown to be efficient also in minimum energy problems over classes of vector measures associated with a standard condenser (see [22]-[26]; see also Remarks 3.1 and 3.2 below for a short survey). The approach developed in [22]-[26] substantially used the assumption of the boundedness of the kernel on the product of the oppositely charged plates of a condenser (see requirement (3.16) below), which made it possible to extend Cartan's proof [5] of the strong completeness of the cone $\mathscr{E}_{\kappa_{2}}^{+}\left(\mathbb{R}^{n}\right)$ of all positive measures on $\mathbb{R}^{n}$ with finite Newtonian energy to an arbitrary perfect kernel $\kappa$ on a locally compact space

\footnotetext{
${ }^{4}$ It follows from Theorem 2.1 that, in fact, for a perfect kernel such a vague cluster point exists and is unique.
} 
$X$ and suitable classes of (signed) measures $\mu \in \mathscr{E}_{K}(X)$; compare with Remark 2.2 above.

\section{Minimum energy problems for a generalized condenser in a locally compact space}

3.1. Vector measures associated with a generalized condenser. A subset $L$ of a topological space $Y$ is said to be locally closed if for every $x \in L$ there is a neighborhood $V$ of $x$ in $Y$ such that $V \cap L$ is a closed subset of the subspace $V$ [2, Chapter I, Section 3, Definition 2], or equivalently if $L$ is the intersection of an open and a closed subset of $Y$ [2, Chapter I, Section 3, Proposition 5].

In a locally compact (Hausdorff) space $X$ we consider an ordered finite collection $\mathbf{A}=\left(A_{i}\right)_{i \in I}$ of nonempty, locally closed sets $A_{i} \subset X$ with the sign $s_{i}:=\operatorname{sign} A_{i}= \pm 1$ prescribed. Denote $I^{+}:=\left\{i \in I: s_{i}=+1\right\}, I^{-}:=I \backslash I^{+}$and $p:=\operatorname{Card} I$, where $p \geqslant 1$ and $I^{-}$is allowed to be empty.

Definition 3.1. We call $\mathbf{A}$ a generalized condenser in $X$ if $A^{+} \cap A^{-}=\varnothing$, where

$$
A^{+}:=\bigcup_{i \in I^{+}} A_{i} \quad \text { and } \quad A^{-}:=\bigcup_{j \in I^{-}} A_{j} .
$$

The sets $A_{i}, i \in I^{+}$, and $A_{j}, j \in I^{-}$, are termed the positive and negative plates of the (generalized) condenser A. To avoid trivialities, we shall always assume that

$$
c_{\kappa}\left(A_{i}\right)>0 \quad \text { for all } i \in I,
$$

the (strictly positive definite) kernel $\kappa$ on $X$ being given. Note that any two equally signed plates may intersect each other or even coincide. Also note that, though $A_{i}$ and $A_{j}$ are disjoint for any $i \in I^{+}$and $j \in I^{-}$, their closures in $X$ may intersect each other even in a set with $c_{\kappa}(\cdot)>0$. The concept of generalized condenser thus defined generalizes that introduced recently in $[14$, Section 3].

Definition 3.2. A generalized condenser $\mathbf{A}$ is said to be standard if all the (locally closed) sets $A_{i}, i \in I$, are closed in $X$.

Unless explicitly stated otherwise, in all that follows we assume $\mathbf{A}$ to be a generalized condenser in $X$. Let $\mathfrak{M}^{+}(\mathbf{A} ; X)$ consist of all positive vector measures $\boldsymbol{\mu}=\left(\mu^{i}\right)_{i \in I}$ where each $\mu^{i}, i \in I$, is a positive scalar Radon measure on $X$ that is carried by $A_{i}$, i.e.

$$
\mathfrak{M}^{+}(\mathbf{A} ; X):=\prod_{i \in I} \mathfrak{M}^{+}\left(A_{i} ; X\right) .
$$


Elements of $\mathfrak{M}^{+}(\mathbf{A} ; X)$ are said to be (vector) measures associated with $\mathbf{A}$. If a measure $\boldsymbol{\mu} \in \mathfrak{M}^{+}(\mathbf{A} ; X)$ and a vector-valued function $\boldsymbol{u}=\left(u_{i}\right)_{i \in I}$ with $\mu^{i}$-measurable components $u_{i}: X \rightarrow[-\infty, \infty]$ are given, then we write

$$
\langle\boldsymbol{u}, \boldsymbol{\mu}\rangle:=\sum_{i \in I}\left\langle u_{i}, \mu^{i}\right\rangle=\sum_{i \in I} \int u_{i} d \mu^{i}
$$

(of course, provided that each $\left\langle u_{i}, \mu^{i}\right\rangle, i \in I$, as well as their sum over $I$ is well defined-as a finite number or $\pm \infty)$.

Being the intersection of an open and a closed subset of $X[2$, Chapter I, Section 3, Proposition 5], each (locally closed) set $A_{i}, i \in I$, is universally measurable, and hence $\mathfrak{M}^{+}\left(A_{i} ; X\right)$ consists of all the restrictions $\left.\mu\right|_{A_{i}}$ where $\mu$ ranges over $\mathfrak{M}^{+}(X)$. On the other hand, according to [2, Chapter I, Section 9, Proposition 13], $A_{i}$ itself can be thought of as a locally compact subspace of $X$. Thus $\mathfrak{M}^{+}\left(A_{i} ; X\right)$ consists, in fact, of all those $v \in \mathfrak{M}^{+}\left(A_{i}\right)$ for each of which there exists $\hat{v} \in \mathfrak{M}^{+}(X)$ with the property

$$
\hat{v}(\varphi)=\left\langle\left.\varphi\right|_{A_{i}}, v\right\rangle \quad \text { for every } \varphi \in C_{0}(X) .
$$

We say that such $\hat{v}$ extends $v \in \mathfrak{M}^{+}\left(A_{i}\right)$ by 0 off $A_{i}$ to all of $X$. A sufficient condition for (3.3) to hold is that $v$ be bounded.

Since $A^{+} \cap A^{-}=\varnothing$, there corresponds to each $\boldsymbol{\mu} \in \mathfrak{M}^{+}(\mathbf{A} ; X)$ a (signed) scalar Radon measure $R_{\mathbf{A}} \boldsymbol{\mu}:=\sum_{i \in I} s_{i} \mu^{i} \in \mathfrak{M}(X)$, the 'resultant' of $\boldsymbol{\mu}$, whose positive and negative parts in the Hahn-Jordan decomposition are given by

$$
\left(R_{\mathbf{A}} \boldsymbol{\mu}\right)^{+}=\sum_{i \in I^{+}} \mu^{i} \quad \text { and } \quad\left(R_{\mathbf{A}} \boldsymbol{\mu}\right)^{-}=\sum_{j \in I^{-}} \mu^{j} .
$$

For the sake of brevity we shall use the short notation $R$ instead of $R_{\mathrm{A}}$ if this will not cause any misunderstanding.

The mapping $\mathfrak{M}^{+}(\mathbf{A} ; X) \ni \boldsymbol{\mu} \mapsto R \boldsymbol{\mu} \in \mathfrak{M}(X)$ is in general non-injective. We shall call $\boldsymbol{\mu}, \boldsymbol{v} \in \mathfrak{M}^{+}(\mathbf{A} ; X) R$-equivalent if $R \boldsymbol{\mu}=R \boldsymbol{v}$. Note that the relation of $R$-equivalence on $\mathfrak{M}^{+}(\mathbf{A} ; X)$ is that of identity $(\boldsymbol{\mu}=\boldsymbol{v})$ if and only if all the $A_{i}, \quad i \in I$, are mutually disjoint. Also observe that $\boldsymbol{\mu} \in \mathfrak{M}^{+}(\mathbf{A} ; X)$ is $R$-equivalent to $\mathbf{0}$ (if and) only if $\boldsymbol{\mu}=\mathbf{0}$.

3.2. A (semi)metric structure on classes of vector measures. For a given (strictly positive definite) kernel $\kappa$ on $X$ and a given (generalized) condenser $\mathbf{A}$, let $\mathscr{E}_{\kappa}^{+}(\mathbf{A} ; X)$ consist of all $\boldsymbol{\mu} \in \mathfrak{M}^{+}(\mathbf{A} ; X)$ such that $\kappa\left(\mu^{i}, \mu^{i}\right)<\infty$ for all $i \in I$; in other words,

$$
\mathscr{E}_{\kappa}^{+}(\mathbf{A} ; X):=\prod_{i \in I} \mathscr{E}_{\kappa}^{+}\left(A_{i} ; X\right)
$$


In view of $\left[11\right.$, Lemma 2.3.1], we see from (3.1) that $\mathscr{E}_{\kappa}^{+}(\mathbf{A} ; X) \neq\{\mathbf{0}\}$; and moreover $\mathscr{E}_{\kappa}^{+}\left(A_{i} ; X\right) \neq\{0\}$ for every $i \in I$.

In accordance with an electrostatic interpretation of a condenser, we say that the interaction between the components $\mu^{i}, i \in I$, of $\boldsymbol{\mu} \in \mathscr{E}_{\kappa}^{+}(\mathbf{A} ; X)$ is determined by the matrix $\left(s_{i} s_{j}\right)_{i, j \in I}$. Given $\boldsymbol{\mu}, \boldsymbol{v} \in \mathscr{E}_{\kappa}^{+}(\mathbf{A} ; X)$, we define the mutual energy

$$
\kappa(\boldsymbol{\mu}, \boldsymbol{v}):=\sum_{i, j \in I} s_{i} s_{j} \kappa\left(\mu^{i}, v^{j}\right)
$$

and the vector potential $\kappa^{\mu}=\left(\kappa^{\mu, i}\right)_{i \in I}$, where

$$
\kappa^{\boldsymbol{\mu}, i}(x):=\sum_{j \in I} s_{i} s_{j} \kappa\left(x, \mu^{j}\right), \quad x \in X .
$$

Lemma 3.1. For any $\boldsymbol{\mu}, \boldsymbol{v} \in \mathscr{E}_{\kappa}^{+}(\mathbf{A} ; X)$ we have

$$
\kappa(\boldsymbol{\mu}, \boldsymbol{v})=\kappa(R \boldsymbol{\mu}, R \boldsymbol{v}) \in(-\infty, \infty) .
$$

Proof. This is obtained directly from (3.4) and (3.5).

For $\boldsymbol{\mu}=\boldsymbol{v} \in \mathscr{E}_{\kappa}^{+}(\mathbf{A} ; X)$ the mutual energy $\kappa(\boldsymbol{\mu}, \boldsymbol{v})$ becomes the energy $\kappa(\boldsymbol{\mu}, \boldsymbol{\mu})$ of $\boldsymbol{\mu}$. Due to the strict positive definiteness of the kernel $\kappa,(3.7)$ yields

$$
\kappa(\boldsymbol{\mu}, \boldsymbol{\mu})=\kappa(R \boldsymbol{\mu}, R \boldsymbol{\mu}) \in[0, \infty) \quad \text { for all } \boldsymbol{\mu} \in \mathscr{E}_{\kappa}^{+}(\mathbf{A} ; X),
$$

where $\kappa(\boldsymbol{\mu}, \boldsymbol{\mu})=0$ if and only if $\boldsymbol{\mu}=\mathbf{0}$.

An assertion $\mathscr{U}(x)$ involving a variable point $x \in X$ is said to hold $c_{\kappa}$-nearly everywhere $\left(c_{\kappa}\right.$-n.e. $)$ on $Q \subset X$ if $c_{\kappa}(N)=0$ where $N$ consists of all $x \in Q$ for which $\mathscr{U}(x)$ fails.

Lemma 3.2. For any $\boldsymbol{\mu} \in \mathscr{E}_{\kappa}^{+}(\mathbf{A} ; X)$ all the $\kappa^{\boldsymbol{\mu}, i}, i \in I$, are well defined and finite $c_{\kappa}$-n.e. on $X$. Moreover,

$$
\kappa^{\boldsymbol{\mu}, i}(\cdot)=s_{i} \kappa(\cdot, R \boldsymbol{\mu}) \quad c_{\kappa} \text {-n.e. on } X .
$$

Proof. Since $\mu^{i} \in \mathscr{E}_{\kappa}^{+}(X)$ for every $i \in I, \kappa\left(\cdot, \mu^{i}\right)$ is finite $c_{\kappa}$-n.e. on $X$ [11, p. 164]. Furthermore, the set of all $x \in X$ with $\kappa\left(x, \mu^{i}\right)=\infty$ is universally measurable, for $\kappa\left(\cdot, \mu^{i}\right)$ is 1.s.c. on $X$. Combined with the fact that the inner capacity $c_{\kappa}(\cdot)$ is subadditive on universally measurable sets [11, Lemma 2.3.5], this implies that $\kappa^{\mu, i}$ is well defined and finite $c_{\kappa}$-n.e. on $X$. Finally, (3.9) is obtained directly from (3.4) and (3.6).

In order to introduce a (semi)metric structure on $\mathscr{E}_{\kappa}^{+}(\mathbf{A} ; X)$, we set

$$
\|\boldsymbol{\mu}-\boldsymbol{v}\|_{\mathscr{E}_{\kappa}^{+}(\mathbf{A} ; X)}:=\|R \boldsymbol{\mu}-R \boldsymbol{v}\|_{\kappa} \quad \text { for all } \boldsymbol{\mu}, \boldsymbol{v} \in \mathscr{E}_{\kappa}^{+}(\mathbf{A} ; X) .
$$


Based on (3.7), we see by straightforward calculation that, in fact,

$$
\|\boldsymbol{\mu}-\boldsymbol{v}\|_{\mathscr{E}_{k}^{+}}^{2}(\mathbf{A} ; X)=\sum_{i, j \in I} s_{i} s_{j} \kappa\left(\mu^{i}-v^{i}, \mu^{j}-v^{j}\right) .
$$

Hence, $\mathscr{E}_{\kappa}^{+}(\mathbf{A} ; X)$ forms a semimetric space with the semimetric $\|\boldsymbol{\mu}-\boldsymbol{v}\|_{\mathscr{E}_{K}^{+}(\mathbf{A} ; X)}$ defined by either of the (equivalent) relations (3.10) or (3.11). Similarly to the terminology for the pre-Hilbert space $\mathscr{E}_{K}(X)$, we therefore call the topology of the semimetric space $\mathscr{E}_{\kappa}^{+}(\mathbf{A} ; X)$ strong. We say that $\boldsymbol{\mu}, \boldsymbol{v} \in \mathscr{E}_{\kappa}^{+}(\mathbf{A} ; X)$ are equivalent in $\mathscr{E}_{\kappa}^{+}(\mathbf{A} ; X)$ if $\|\boldsymbol{\mu}-\boldsymbol{v}\|_{\mathscr{E}_{K}^{+}(\mathbf{A} ; X)}=0$, or equivalently if $R \boldsymbol{\mu}=R \boldsymbol{v}$, the latter being clear from (3.10) in view of the strict positive definiteness of the kernel.

LeMma 3.3. The relation of equivalence on $\mathscr{E}_{\kappa}^{+}(\mathbf{A} ; X)$ amounts to that of identity if and only if all the $A_{i}, i \in I$, are mutually essentially disjoint, i.e. with $c_{\kappa}\left(A_{i} \cap A_{j}\right)=0$ for all $i \neq j$.

Proof. Since a nonzero positive scalar measure of finite energy does not charge any set of zero capacity [11, Lemma 2.3.1], the sufficiency part of the lemma is obvious. To prove the necessity part, assume on the contrary that there are two equally signed plates $A_{k}$ and $A_{\ell}, k \neq \ell$, with $c_{k}\left(A_{k} \cap A_{\ell}\right)$ $>0$. By [11, Lemma 2.3.1], there exists a nonzero positive scalar measure $\tau \in \mathscr{E}_{\kappa}^{+}\left(A_{k} \cap A_{\ell}\right)$. Choose $\boldsymbol{\mu}=\left(\mu^{i}\right)_{i \in I} \in \mathscr{E}_{\kappa}^{+}(\mathbf{A})$ such that $\left.\mu^{k}\right|_{A_{k} \cap A_{\ell}}-\tau \geqslant 0$, and define $\boldsymbol{\mu}_{m}=\left(\mu_{m}^{i}\right)_{i \in I} \in \mathscr{E}_{K}^{+}(\mathbf{A}), m=1,2$, where $\mu_{1}^{k}:=\mu^{k}-\tau$ and $\mu_{1}^{i}:=\mu^{i}$ for all $i \neq k$, while $\mu_{2}^{\ell}:=\mu^{\ell}+\tau$ and $\mu_{2}^{i}:=\mu^{i}$ for all $i \neq \ell$. Then $R \boldsymbol{\mu}_{1}=R \boldsymbol{\mu}_{2}$, and hence $\boldsymbol{\mu}_{1}$ and $\boldsymbol{\mu}_{2}$ are equivalent in $\mathscr{E}_{\kappa}^{+}(\mathbf{A} ; X)$, but $\boldsymbol{\mu}_{1} \neq \boldsymbol{\mu}_{2}$.

Summarizing what we have observed, we are led to the following conclusion.

THEOREM 3.1. $\mathscr{E}_{K}^{+}(\mathbf{A} ; X)$ is a semimetric space with the semimetric defined by either of the (equivalent) relations (3.10) or (3.11), and this space is isometric to its $R$-image in $\mathscr{E}_{K}(X)$. The semimetric $\|\boldsymbol{\mu}-\boldsymbol{v}\|_{\mathscr{E}_{\kappa}^{+}(\mathbf{A} ; X)}$ is actually a metric if and only if all the $A_{i}, i \in I$, are mutually essentially disjoint.

3.3. The vague topology on $\mathfrak{M}^{+}(\mathbf{A} ; X)$. In Section 3.3 we consider a standard condenser A (see Definition 3.2). The set of all (vector) measures associated with $\mathbf{A}$ can be endowed with the vague topology defined as follows.

Definition 3.3. The vague topology on $\mathfrak{M}^{+}(\mathbf{A} ; X), \mathbf{A}=\left(A_{i}\right)_{i \in I}$ being a standard condenser in $X$, is the topology of the product space $\prod_{i \in I} \mathfrak{M i}^{+}\left(A_{i} ; X\right)$ where each of the $\mathfrak{M}^{+}\left(A_{i} ; X\right)$ is endowed with the vague topology induced from $\mathfrak{M}(X)$. Namely, a sequence $\left\{\boldsymbol{\mu}_{k}\right\}_{k \in \mathbb{N}} \subset \mathfrak{M}^{+}(\mathbf{A} ; X)$ converges to $\boldsymbol{\mu} \in \mathfrak{M}^{+}(\mathbf{A} ; X)$ vaguely if for every $i \in I, \mu_{k}^{i} \rightarrow \mu^{i}$ vaguely in $\mathfrak{M}(X)$ as $k \rightarrow \infty$. 
Since all the $A_{i}, i \in I$, are closed in $X, \mathfrak{M}^{+}(\mathbf{A} ; X)$ is vaguely closed in $\mathfrak{M}(X)^{p}$. Besides, since every $\mathfrak{M}^{+}\left(A_{i} ; X\right)$ is Hausdorff in the vague topology, so is $\mathfrak{M}^{+}(\mathbf{A} ; X)$ [2, Chapter I, Section 8 , Proposition 7]. Hence, a vague limit of any sequence in $\mathfrak{M}^{+}(\mathbf{A} ; X)$ belongs to $\mathfrak{M}^{+}(\mathbf{A} ; X)$ and is unique (whenever it exists). We call a set $\mathfrak{F} \subset \mathfrak{M}^{+}(\mathbf{A} ; X)$ vaguely bounded if for every $\varphi \in C_{0}(X)$,

$$
\sup _{\boldsymbol{\mu} \in \mathscr{F}}\left|\mu^{i}(\varphi)\right|<\infty \quad \text { for all } i \in I .
$$

LeMma 3.4. A vaguely bounded set $\mathfrak{F} \subset \mathfrak{M}^{+}(\mathbf{A} ; X)$ is vaguely relatively compact.

Proof. It is clear from the above definition that for every $i \in I$ the set

$$
\mathfrak{F}^{i}:=\left\{\mu^{i} \in \mathfrak{M}^{+}\left(A_{i} ; X\right): \boldsymbol{\mu}=\left(\mu^{j}\right)_{j \in I} \in \mathfrak{F}\right\}
$$

is vaguely bounded in $\mathfrak{M}^{+}(X)$; and hence, by [3, Chapter III, Section 2, Proposition 9], $\mathfrak{F}^{i}$ is vaguely relatively compact in $\mathfrak{M}(X)$. Since $\mathfrak{F} \subset \prod_{i \in I} \mathfrak{F}^{i}$, the lemma follows from Tychonoff's theorem on the product of compact spaces [2, Chapter I, Section 9, Theorem 3].

\subsection{An unconstrained weighted minimum energy problem for vector measures.}

Let a (generalized) condenser $\mathbf{A}=\left(A_{i}\right)_{i \in I}$ and a (strictly positive definite) kernel $\kappa$ on $X$ be given. Fix a vector-valued function $\mathbf{f}=\left(f_{i}\right)_{i \in I}$, where each $f_{i}: X \rightarrow[-\infty, \infty]$ is $\mu$-measurable for every $\mu \in \mathscr{E}_{\kappa}^{+}\left(A_{i} ; X\right)$ and $f_{i}$ is treated as an external field acting on the charges (measures) from $\mathscr{E}_{\kappa}^{+}\left(A_{i} ; X\right)$. The f-weighted vector potential and the f-weighted energy of $\boldsymbol{\mu} \in \mathscr{E}_{\kappa}^{+}(\mathbf{A} ; X)$ are (formally) defined by

$$
\begin{aligned}
\mathbf{W}_{\kappa, \mathbf{f}}^{\mu} & :=\kappa^{\mu}+\mathbf{f}, \\
G_{\kappa, \mathbf{f}}(\boldsymbol{\mu}) & :=\kappa(\boldsymbol{\mu}, \boldsymbol{\mu})+2\langle\mathbf{f}, \boldsymbol{\mu}\rangle,
\end{aligned}
$$

respectively. ${ }^{5}$ Thus $\mathbf{W}_{\kappa, \mathbf{f}}^{\boldsymbol{\mu}}=\left(W_{\kappa, \mathbf{f}}^{\boldsymbol{\mu}, i}\right)_{i \in I}$, where $W_{\kappa, \mathbf{f}}^{\boldsymbol{\mu}, i}:=\kappa^{\boldsymbol{\mu}, i}+f_{i}$ (see (3.6)).

Let $\mathscr{E}_{\kappa, \mathbf{f}}^{+}(\mathbf{A} ; X)$ consist of all $\boldsymbol{\mu} \in \mathscr{E}_{\kappa}^{+}(\mathbf{A} ; X)$ such that each $f_{i}, i \in I$, is $\mu^{i}$-integrable. For every $\boldsymbol{\mu} \in \mathscr{E}_{\kappa}^{+}(\mathbf{A} ; X)$, both $G_{\kappa, \mathbf{f}}(\boldsymbol{\mu})$ and $\langle\mathbf{f}, \boldsymbol{\mu}\rangle$ are finite.

Lemma 3.5. Suppose that a set $\mathfrak{E} \subset \mathscr{E}_{\kappa, \mathbf{f}}^{+}(\mathbf{A} ; X)$ is convex. Then there exists $\lambda \in \mathbb{E}$ with

$$
G_{\kappa, \mathbf{f}}(\lambda)=\min _{\boldsymbol{\mu} \in \mathfrak{E}} G_{\kappa, \mathbf{f}}(\boldsymbol{\mu})
$$

\footnotetext{
${ }^{5} G_{\kappa, \mathbf{f}}(\cdot)$ is also known as the Gauss functional (see e.g. [21]; compare with [17]). Note that when defining $G_{\kappa, \mathbf{f}}(\cdot)$, we have used the notation (3.2).
} 
if and only if

$$
\sum_{i \in I}\left\langle W_{\kappa, \mathbf{f}}^{\lambda, i}, \mu^{i}-\lambda^{i}\right\rangle \geqslant 0 \quad \text { for all } \boldsymbol{\mu} \in \mathbb{E} .
$$

Proof. By direct calculation, for any $\boldsymbol{\mu}, \boldsymbol{v} \in \mathfrak{E}$ and any $h \in(0,1]$ we get

$$
G_{\kappa, \mathbf{f}}(h \boldsymbol{\mu}+(1-h) \boldsymbol{v})-G_{\kappa, \mathbf{f}}(\boldsymbol{v})=2 h \sum_{i \in I}\left\langle W_{\kappa, \mathbf{f}}^{\boldsymbol{v}, i}, \mu^{i}-v^{i}\right\rangle+h^{2}\|\boldsymbol{\mu}-\boldsymbol{v}\|_{\mathscr{E}_{k}^{+}}^{2}(\mathbf{A} ; X) .
$$

If $\boldsymbol{v}=\boldsymbol{\lambda}$ satisfies (3.14), then the left (hence, also the right) side of this display is $\geqslant 0$, which leads to (3.15) by letting $h \rightarrow 0$. Conversely, if (3.15) holds, then the preceding formula with $\boldsymbol{v}=\lambda$ and $h=1$ gives $G_{\kappa, \mathbf{f}}(\boldsymbol{\mu}) \geqslant G_{\kappa, \mathbf{f}}(\lambda)$ for all $\boldsymbol{\mu} \in \mathfrak{E}$, and (3.14) follows.

Fix a numerical vector $\mathbf{a}=\left(a_{i}\right)_{i \in I}$ with $0<a_{i}<\infty, i \in I$, and write

$$
\begin{aligned}
\mathfrak{M}^{+}(\mathbf{A}, \mathbf{a} ; X) & :=\left\{\boldsymbol{\mu} \in \mathfrak{M}^{+}(\mathbf{A} ; X): \mu^{i}\left(A_{i}\right)=a_{i} \text { for all } i \in I\right\}, \\
\mathscr{E}_{\kappa}^{+}(\mathbf{A}, \mathbf{a} ; X) & :=\mathscr{E}_{\kappa}^{+}(\mathbf{A} ; X) \cap \mathfrak{M}^{+}(\mathbf{A}, \mathbf{a} ; X), \\
\mathscr{E}_{\kappa, \mathbf{f}}^{+}(\mathbf{A}, \mathbf{a} ; X) & :=\mathscr{E}_{\kappa, \mathbf{f}}^{+}(\mathbf{A} ; X) \cap \mathfrak{M}^{+}(\mathbf{A}, \mathbf{a} ; X) .
\end{aligned}
$$

If the class $\mathscr{E}_{\kappa, \mathbf{f}}^{+}(\mathbf{A}, \mathbf{a} ; X)$ is nonempty, or equivalently if

$$
G_{\kappa, \mathbf{f}}(\mathbf{A}, \mathbf{a} ; X):=\inf _{\boldsymbol{\mu} \in \mathscr{E}_{\kappa, \mathbf{f}}^{+}(\mathbf{A}, \mathbf{a} ; X)} G_{\kappa, \mathbf{f}}(\boldsymbol{\mu})<\infty,
$$

then the following unconstrained weighted minimum energy problem makes sense.

Problem 3.1. Given $X, \kappa, \mathbf{A}$, a and $\mathbf{f}$, does there exist $\lambda_{\mathbf{A}} \in \mathscr{E}_{\kappa, \mathbf{f}}^{+}(\mathbf{A}, \mathbf{a} ; X)$ with $G_{\kappa, \mathbf{f}}\left(\lambda_{\mathbf{A}}\right)=G_{\kappa, \mathbf{f}}(\mathbf{A}, \mathbf{a} ; X)$ ?

If $I=I^{+}=\{1\}, A_{1}$ is closed, $a_{1}=1$ and $f_{1}=0$, then Problem 3.1 reduces to problem (2.1), solved in [11, Theorem 4.1] (see Remark 2.3 above).

REMARK 3.1. Let $\mathbf{A}$ be a standard condenser in $X$ such that

$$
\sup _{(x, y) \in A^{+} \times A^{-}} \kappa(x, y)<\infty .
$$

Under these assumptions, in [25, 26] an approach has been worked out based on both the vague and the strong topologies on $\mathscr{E}_{\kappa}^{+}(\mathbf{A} ; X)$ which made it possible to provide a fairly complete analysis of Problem 3.1. In more detail, it was shown that if the kernel $\kappa$ is perfect and if for all $i \in I$ either $f_{i} \in \Psi(X)$ or $f_{i}=s_{i} \kappa(\cdot, \zeta)$ for some (signed) $\zeta \in \mathscr{E}_{\kappa}(X)$, then the requirement

$$
c_{\kappa}\left(A^{+} \cup A^{-}\right)<\infty
$$


is sufficient for Problem 3.1 to be solvable for every vector a [25, Theorem 8.1]. However, if (3.17) fails, then in general there exists a vector $\mathbf{a}^{\prime}$ such that the problem has no solution (see [25]). ${ }^{6}$ Therefore, it was interesting to give a description of the set of all vectors a for which Problem 3.1 is nevertheless solvable. Such a characterization has been established in [26] (cf. the footnote to Remark 3.2 below). On the other hand, if assumption (3.16) is omitted, then the approach developed in $[25,26]$ breaks down, and (3.17) does not guarantee anymore the existence of a solution to Problem 3.1. This has been illustrated by [14, Theorem 4.6], pertaining to the Newtonian kernel $|x-y|^{2-n}$ on $\mathbb{R}^{n}, n \geqslant 3$.

3.5. A constrained weighted minimum energy problem for vector measures. A measure $\sigma^{i} \in \mathfrak{M}^{+}\left(A_{i} ; X\right)$ is said to be a constraint for elements of $\mathfrak{M i}^{+}\left(A_{i}, a_{i} ; X\right)$ if $\sigma^{i}\left(A_{i}\right)>a_{i}$. Let $\mathfrak{C}\left(A_{i} ; X\right)$ consist of all these $\sigma^{i}$, and let

$$
\mathfrak{C}(\mathbf{A} ; X):=\prod_{i \in I} \mathfrak{C}\left(A_{i} ; X\right) .
$$

Consider $\xi=\left(\xi^{i}\right)_{i \in I}$ such that for each $i \in I$ either $\xi^{i}=\sigma^{i} \in \mathbb{C}\left(A_{i} ; X\right)$ or $\xi^{i}=\infty$, where the formal notation $\xi^{i}=\infty$ means that no upper constraint on the elements of $\mathfrak{M}^{+}\left(A_{i}, a_{i} ; X\right)$ is imposed, and define

$$
\mathfrak{M}^{\xi}(\mathbf{A} ; X):=\left\{\boldsymbol{\mu} \in \mathfrak{M}^{+}(\mathbf{A} ; X): \mu^{i} \leqslant \xi^{i} \text { for all } i \in I\right\} .
$$

(In the case where $\xi^{i}=\sigma^{i} \in \mathfrak{C}\left(A_{i} ; X\right)$ the expression $\mu^{i} \leqslant \xi^{i}$ means that the measure $\xi^{i}-\mu^{i}$ is positive, while we are making the obvious convention that any positive scalar Radon measure is $<\infty$.) Also write

$$
\begin{aligned}
& \mathfrak{M}^{\xi}(\mathbf{A}, \mathbf{a} ; X):=\mathfrak{M}^{+}(\mathbf{A}, \mathbf{a} ; X) \cap \mathfrak{M}^{\xi}(\mathbf{A} ; X), \\
& \mathscr{E}_{\kappa}^{\xi}(\mathbf{A}, \mathbf{a} ; X):=\mathscr{E}_{\kappa}^{+}(\mathbf{A}, \mathbf{a} ; X) \cap \mathfrak{M}^{\xi}(\mathbf{A} ; X), \\
& \mathscr{E}_{\kappa, \mathbf{f}}^{\xi}(\mathbf{A}, \mathbf{a} ; X):=\mathscr{E}_{\kappa, \mathbf{f}}^{+}(\mathbf{A}, \mathbf{a} ; X) \cap \mathfrak{M}^{\xi}(\mathbf{A} ; X) .
\end{aligned}
$$

If the class $\mathscr{E}_{\kappa, \mathbf{f}}^{\xi}(\mathbf{A}, \mathbf{a} ; X)$ is nonempty, or equivalently if

$$
G_{\kappa, \mathbf{f}}^{\xi}(\mathbf{A}, \mathbf{a} ; X):=\inf _{\boldsymbol{\mu} \in \mathscr{E}_{\kappa, \mathbf{f}}^{\xi}(\mathbf{A}, \mathbf{a} ; X)} G_{\kappa, \mathbf{f}}(\boldsymbol{\mu})<\infty,
$$

then the following constrained weighted minimum energy problem makes sense.

\footnotetext{
${ }^{6}$ In the case of the $\alpha$-Riesz kernels of order $1<\alpha \leqslant 2$ on $\mathbb{R}^{3}$, some of the (theoretical) results on the solvability or unsolvability of Problem 3.1 established in [25] have been illustrated in [18, 20] by means of numerical experiments.
} 
Problem 3.2. Given $X, \kappa, \mathbf{A}, \mathbf{a}, \mathbf{f}$ and $\boldsymbol{\xi}$, does there exist a vector measure $\lambda_{\mathbf{A}}^{\xi} \in \mathscr{E}_{\kappa, \mathbf{f}}^{\xi}(\mathbf{A}, \mathbf{a} ; X)$ with $G_{\kappa, \mathbf{f}}\left(\lambda_{\mathbf{A}}^{\xi}\right)=G_{\kappa, \mathbf{f}}^{\xi}(\mathbf{A}, \mathbf{a} ; X)$ ?

REMARK 3.2. Assume for a moment that (3.16) holds, the condenser A is standard, the kernel $\kappa$ is perfect, and the external field $\mathbf{f}$ is as described in Remark 3.1. It has been shown in [24, Theorem 6.2] that condition (3.17) then guarantees the existence of a solution to Problem 3.2 for any $\xi$ and any vector a. $^{7}$ Recently it has been shown by the present authors [14, Theorem 6.1(i)] that this assertion on the solvability of Problem 3.2 remains valid if instead of (3.17) it is assumed that $\boldsymbol{\xi}=\boldsymbol{\sigma} \in \mathfrak{C}(\mathbf{A} ; X)$ is bounded, i.e. with $\sum_{i \in I} \sigma^{i}\left(A_{i}\right)<\infty$.

REMARK 3.3. In our recent work [14], Problem 3.2 has also been analyzed for an ordered finite collection of compact sets with the sign $s_{i}= \pm 1$ prescribed such that oppositely charged sets intersect each other in a set of zero $\kappa$-capacity, $\kappa$ being any positive definite (not necessarily perfect) kernel. It has been shown that if $\kappa$ is $\operatorname{regular}^{8}$ and each of the potentials $\kappa\left(\cdot, \xi^{i}\right)$ is (finitely) continuous on the support $S\left(\xi^{i}\right)$, then a solution to Problem 3.2 exists [14, Theorems 6.1(ii)].

Let $\boldsymbol{\Xi}_{\kappa, \mathbf{f}}^{\xi}(\mathbf{A}, \mathbf{a} ; X)$ consist of all solutions to Problem 3.2 (if these exist).

Lemma 3.6. Any two elements $\lambda, \breve{\lambda} \in \Xi_{\kappa, \mathbf{f}}^{\xi}(\mathbf{A}, \mathbf{a} ; X)$ are R-equivalent.

Proof. This can be established by standard methods based on the convexity of the class $\mathscr{E}_{\kappa, \mathbf{f}}^{\xi}(\mathbf{A}, \mathbf{a} ; X)$, the isometry between this class and its $R$-image in $\mathscr{E}_{K}(X)$, and the pre-Hilbert structure on the space $\mathscr{E}_{K}(X)$. Indeed, in view of the convexity of $\mathscr{E}_{\kappa, \mathbf{f}}^{\xi}(\mathbf{A}, \mathbf{a} ; X)$, relations (3.8) and (3.13) imply

$$
4 G_{\kappa, \mathbf{f}}^{\xi}(\mathbf{A}, \mathbf{a} ; X) \leqslant 4 G_{\kappa, \mathbf{f}}\left(\frac{\lambda+\breve{\lambda}}{2}\right)=\|R \lambda+R \breve{\lambda}\|_{\kappa}^{2}+4\langle\mathbf{f}, \lambda+\breve{\lambda}\rangle .
$$

On the other hand, applying the parallelogram identity in $\mathscr{E}_{K}(X)$ to $R \lambda$ and $R \breve{\lambda}$ and then adding and subtracting $4\langle\mathbf{f}, \lambda+\breve{\lambda}\rangle$ we get

$$
\|R \lambda-R \breve{\lambda}\|_{\kappa}^{2}=-\|R \lambda+R \breve{\lambda}\|_{\kappa}^{2}-4\langle\mathbf{f}, \lambda+\breve{\lambda}\rangle+2 G_{\kappa, \mathbf{f}}(\lambda)+2 G_{\kappa, \mathbf{f}}(\breve{\lambda}) .
$$

When combined with the preceding relation, this yields

$$
0 \leqslant\|R \lambda-R \breve{\lambda}\|_{\kappa}^{2} \leqslant-4 G_{\kappa, \mathbf{f}}^{\xi}(\mathbf{A}, \mathbf{a} ; X)+2 G_{\kappa, \mathbf{f}}(\lambda)+2 G_{\kappa, \mathbf{f}}(\breve{\lambda})=0,
$$

which establishes the lemma because of the strict positive definiteness of $\kappa$.

\footnotetext{
${ }^{7}$ Actually, this result and those described in Remark 3.1 have been obtained in [24]-[26] even for infinite dimensional vector measures.

${ }^{8} \mathrm{~A}$ kernel $\kappa$ is said to be regular if for any $\mu \in \mathfrak{M}^{+}(X)$ with compact $S(\mu)$, the potential $\kappa(\cdot, \mu)$ is continuous throughout $X$ whenever its restriction to $S(\mu)$ is continuous, see e.g. [21].
} 
COROllary 3.1. If the class $\mathfrak{S}_{\kappa, \mathbf{f}}^{\xi}(\mathbf{A}, \mathbf{a} ; X)$ is nonempty, then it reduces to a single element whenever all the $A_{i}, i \in I$, are mutually essentially disjoint.

Proof. This is obtained directly from Lemmas 3.6 and 3.3.

\section{4. $\alpha$-Riesz balayage and $\alpha$-Green kernel}

In all that follows fix $n \geqslant 3, \alpha \in(0,2]$ and a domain $D \subset \mathbb{R}^{n}$ with $c_{\kappa_{\alpha}}\left(D^{c}\right)>0$, where $D^{c}:=\mathbb{R}^{n} \backslash D$, and assume that either $\kappa(x, y)=\kappa_{\alpha}(x, y):=$ $|x-y|^{\alpha-n}$ is the $\alpha$-Riesz kernel on $X=\mathbb{R}^{n}$, or $\kappa(x, y)=g_{D}^{\alpha}(x, y)$ is the $\alpha$-Green kernel on $X=D$. For the definition of $g_{D}^{\alpha}$, see [19, Chapter IV, Section 5] or see below.

For given $x \in \mathbb{R}^{n}$ and $r \in(0, \infty)$ write $B(x, r):=\left\{y \in \mathbb{R}^{n}:|y-x|<r\right\}$, $S(x, r):=\left\{y \in \mathbb{R}^{n}:|y-x|=r\right\}$ and $\bar{B}(x, r):=B(x, r) \cup S(x, r)$.

We simply write $\alpha$ instead of $\kappa_{\alpha}$ if $\kappa_{\alpha}$ serves as an index, and we use the short form 'n.e.' instead of ' $c_{\alpha}$-n.e.' if this will not cause any misunderstanding. When speaking of a positive scalar Radon measure $\mu \in \mathfrak{M}^{+}\left(\mathbb{R}^{n}\right)$, we always assume that for the given $\alpha, \kappa_{\alpha} \mu$ is not identically infinite. This implies that

$$
\int_{|y|>1} \frac{d \mu(y)}{|y|^{n-\alpha}}<\infty
$$

(see [19, Eq. 1.3.10]), and consequently that $\kappa_{\alpha}(\cdot, \mu)$ is finite $\left(c_{\alpha^{-}}\right)$n.e. on $\mathbb{R}^{n}[19$, Chapter III, Section 1]; these two implications can actually be reversed.

Definition 4.1. A (signed) measure $v \in \mathfrak{M}(D)$ is called extendible if there exist $\widehat{v^{+}}$and $\widehat{v^{-}}$extending $v^{+}$and $v^{-}$, respectively, by 0 off $D$ to $\mathbb{R}^{n}$ (see (3.3)), and if these $\widehat{v^{+}}$and $\widehat{v^{-}}$satisfy (4.1). We identify such $v \in \mathfrak{M}(D)$ with its extension $\hat{v}:=\widehat{v^{+}}-\widehat{v^{-}}$, and we therefore write $\hat{v}=v$.

Every bounded measure $v \in \mathfrak{M}(D)$ is extendible. The converse holds if $D$ is bounded, but not in general (e.g. not if $D^{c}$ is compact). The set of all extendible measures consists of all the restrictions $\left.\mu\right|_{D}$ where $\mu$ ranges over $\mathfrak{M}\left(\mathbb{R}^{n}\right)$.

The $\alpha$-Green kernel $g=g_{D}^{\alpha}$ on $D$ is defined by

$$
g_{D}^{\alpha}(x, y)=\kappa_{\alpha}\left(x, \varepsilon_{y}\right)-\kappa_{\alpha}\left(x, \varepsilon_{y}^{D^{c}}\right) \quad \text { for all } x, y \in D,
$$

where $\varepsilon_{y}$ denotes the unit Dirac measure at a point $y$ and $\varepsilon_{y}^{D^{c}}$ its $\alpha$-Riesz balayage onto the (closed) set $D^{c}$, determined uniquely in the frame of the classical approach by [15, Theorem 3.6] pertaining to positive Radon measures on $\mathbb{R}^{n}$. See also the book by Bliedtner and Hansen [1] where balayage is studied in the setting of balayage spaces. 
We shall simply write $\mu^{\prime}$ instead of $\mu^{D^{c}}$ when speaking of the $\alpha$-Riesz balayage of $\mu \in \mathfrak{M}^{+}\left(D ; \mathbb{R}^{n}\right)$ onto $D^{c}$. According to [15, Corollaries 3.19, 3.20], for any $\mu \in \mathfrak{M}^{+}\left(D ; \mathbb{R}^{n}\right)$ the balayage $\mu^{\prime}$ is $c_{\alpha}$-absolutely continuous and it is determined uniquely by relation

$$
\kappa_{\alpha}\left(\cdot, \mu^{\prime}\right)=\kappa_{\alpha}(\cdot, \mu) \quad \text { n.e. on } D^{c}
$$

among the $c_{\alpha}$-absolutely continuous measures supported by $D^{c}$. Furthermore, there holds the integral representation ${ }^{9}$

$$
\mu^{\prime}=\int \varepsilon_{y}^{\prime} d \mu(y)
$$

(see [15, Theorem 3.17]). If moreover $\mu \in \mathscr{E}_{\alpha}^{+}\left(D ; \mathbb{R}^{n}\right)$, then the balayage $\mu^{\prime}$ is in fact the orthogonal projection of $\mu$ onto the convex cone $\mathscr{E}_{\alpha}^{+}\left(D^{c} ; \mathbb{R}^{n}\right)$ (see $[13$, Theorem 4.12] or [15, Theorem 3.1]), i.e. $\mu^{\prime} \in \mathscr{E}_{\alpha}^{+}\left(D^{c} ; \mathbb{R}^{n}\right)$ and

$$
\|\mu-\theta\|_{\alpha}>\left\|\mu-\mu^{\prime}\right\|_{\alpha} \quad \text { for all } \theta \in \mathscr{E}_{\alpha}^{+}\left(D^{c} ; \mathbb{R}^{n}\right), \theta \neq \mu^{\prime} .
$$

If now $v \in \mathfrak{M}(D)$ is an extendible (signed) measure, then

$$
v^{\prime}:=v^{D^{c}}:=\left(v^{+}\right)^{\prime}-\left(v^{-}\right)^{\prime}
$$

is said to be a balayage of $v$ onto $D^{c}$. It follows from [19, Chapter III, Section $1, \mathrm{n}^{\circ} 1$, Remark] that the balayage $v^{\prime}$ is determined uniquely by (4.2) with $v$ in place of $\mu$ among the $c_{\alpha}$-absolutely continuous measures supported by $D^{c}$.

The following definition goes back to Brelot [4, Theorem VII.13].

Definition 4.2. A closed set $F \subset \mathbb{R}^{n}$ is said to be $\alpha$-thin at infinity if either $F$ is compact, or the inverse of $F$ relative to $S(0,1)$ has $x=0$ as an $\alpha$-irregular boundary point (cf. [19, Theorem 5.10]).

THEOREM 4.1 (see [15, Theorem 3.22]). The set $D^{c}$ is not $\alpha$-thin at infinity if and only if for every bounded measure $\mu \in \mathfrak{M}^{+}(D)$ we have

$$
\mu^{\prime}\left(\mathbb{R}^{n}\right)=\mu\left(\mathbb{R}^{n}\right) .
$$

As noted in Remark 2.1 above, the $\alpha$-Riesz kernel $\kappa_{\alpha}$ on $\mathbb{R}^{n}$ and the $\alpha$-Green kernel $g_{D}^{\alpha}$ on $D$ are both strictly positive definite and moreover perfect. Furthermore, the $\alpha$-Riesz kernel $\kappa_{\alpha}$ (with $\alpha \in(0,2]$ ) satisfies the complete

\footnotetext{
${ }^{9}$ In the literature the integral representation (4.3) seems to have been more or less taken for granted, though it has been pointed out in [3, Chapter V, Section $\left.3, n^{\circ} 1\right]$ that it requires that the family $\left(\varepsilon_{y}^{\prime}\right)_{y \in D}$ be $\mu$-adequate in the sense of [3, Chapter V, Section 3, Definition 1] (see also counterexamples (without $\mu$-adequacy) in Exercises 1 and 2 at the end of that section). A proof of this adequacy has therefore been given in [15, Lemma 3.16].
} 
maximum principle in the form stated in Section 2 (see [19, Theorems 1.27, 1.29]). Regarding a similar result for the $\alpha$-Green kernel $g$, the following assertion holds.

TheOrem 4.2 (see [15, Theorem 4.6]). Let $\mu \in \mathscr{E}_{g}^{+}(D)$, let $v \in \mathfrak{M}^{+}(D)$ be extendible, and let $w$ be a positive $\alpha$-superharmonic function on $\mathbb{R}^{n}[19$, Chapter I, Section 5, $n^{\circ}$ 20]. If moreover $g(\cdot, \mu) \leqslant g(\cdot, v)+w(\cdot) \mu$-a.e. on $D$, then the same inequality holds on all of $D$.

The following three lemmas establish relations between potentials and energies relative to the kernels $\kappa_{\alpha}$ and $g=g_{D}^{\alpha}$.

Lemma 4.1. For any extendible measure $\mu \in \mathfrak{M}(D)$ the $\alpha$-Green potential $g(\cdot, \mu)$ is finite $\left(c_{\alpha^{-}}\right)$n.e. on $D$ and given by

$$
g(\cdot, \mu)=\kappa_{\alpha}\left(\cdot, \mu-\mu^{\prime}\right) \quad \text { n.e. on } D .
$$

Proof. It is seen from Definition 4.1 that $\kappa_{\alpha}(\cdot, \mu)$ is (well defined and) finite n.e. on $\mathbb{R}^{n}$, and hence so is $\kappa_{\alpha}\left(\cdot, \mu^{\prime}\right)$. Applying (4.3) to $\mu^{ \pm}$, we get by [3, Chapter V, Section 3, Theorem 1]

$$
g(\cdot, \mu)=\int\left[\kappa_{\alpha}\left(\cdot, \varepsilon_{y}\right)-\kappa_{\alpha}\left(\cdot, \varepsilon_{y}^{\prime}\right)\right] d \mu(y)=\kappa_{\alpha}(\cdot, \mu)-\kappa_{\alpha}\left(\cdot, \mu^{\prime}\right)
$$

n.e. on $D$, as was to be proved.

REMARK 4.1. Lemma 4.1 is valid with ' $c_{g}$-n.e.' in place of ' $\left(c_{\alpha^{-}}\right)$n.e.' since, by [9, Lemma 2.6], for any $Q \subset D, c_{g}(Q)=0 \Leftrightarrow c_{\alpha}(Q)=0$.

Lemma 4.2. Suppose that $\mu \in \mathfrak{M}(D)$ is extendible and the extension belongs to $\mathscr{E}_{\alpha}\left(\mathbb{R}^{n}\right)$. Then

$$
\begin{aligned}
& \mu \in \mathscr{E}_{g}(D), \\
& \mu-\mu^{\prime} \in \mathscr{E}_{\alpha}\left(\mathbb{R}^{n}\right), \\
& \|\mu\|_{g}^{2}=\left\|\mu-\mu^{\prime}\right\|_{\alpha}^{2}=\|\mu\|_{\alpha}^{2}-\left\|\mu^{\prime}\right\|_{\alpha}^{2} .
\end{aligned}
$$

Proof. In view of the definition of a (signed) measure of finite energy (see Section 2), we obtain (4.7) from the inequality ${ }^{10}$

$$
g_{D}^{\alpha}(x, y)<\kappa_{\alpha}(x, y) \quad \text { for all } x, y \in D,
$$

while (4.8) from [15, Corollary 3.7] (or [15, Theorems 3.1 and 3.6]). According to Lemma 4.1 and Remark $4.1, g(\cdot, \mu)$ is finite $c_{g}$-n.e. on $D$ and given by (4.6),

\footnotetext{
${ }^{10}$ The strict inequality in $(4.10)$ is caused by our convention $c_{\alpha}\left(D^{c}\right)>0$.
} 
while by (4.7) the same holds $|\mu|$-a.e. on $D$ (see [11, Lemma 2.3.1]). Integrating (4.6) with respect to $\mu^{ \pm}$, we therefore get by subtraction

$$
\infty>g(\mu, \mu)=\kappa_{\alpha}\left(\mu-\mu^{\prime}, \mu\right) .
$$

Since $\kappa_{\alpha}\left(\cdot, \mu-\mu^{\prime}\right)=0$ n.e. on $D^{c}$ by (4.2), we see from the $c_{\alpha}$-absolute continuity of $\mu^{\prime}$ that

$$
\kappa_{\alpha}\left(\mu-\mu^{\prime}, \mu^{\prime}\right)=0
$$

which results in the former equality in (4.9) when combined with (4.11). Because of (4.8), relation (4.12) takes the form $\left\|\mu^{\prime}\right\|_{\alpha}^{2}=\kappa_{\alpha}\left(\mu, \mu^{\prime}\right)$, and the former equality in (4.9) implies the latter.

LemmA 4.3. Assume that $\mu \in \mathfrak{M}(D)$ has compact support $S_{D}^{\mu}$. Then $\mu \in \mathscr{E}_{g}(D)$ if and only if its extension belongs to $\mathscr{E}_{\alpha}\left(\mathbb{R}^{n}\right) .{ }^{11}$

Proof. According to Lemma 4.2, it is enough to establish the 'only if' part of the lemma. We may clearly assume that $\mu$ is positive. Since $\kappa_{\alpha}\left(\cdot, \mu^{\prime}\right)$ is continuous on $D$ and hence bounded on the compact set $S_{D}^{\mu}$, we have

$$
\kappa_{\alpha}\left(\mu, \mu^{\prime}\right)<\infty .
$$

But $g(\mu, \mu)$ is finite by assumption, and therefore likewise as in the preceding proof relation (4.11) holds. Combining (4.11) with (4.13) yields $\mu \in \mathscr{E}_{\alpha}\left(\mathbb{R}^{n}\right)$.

\section{Minimum $\alpha$-Riesz energy problems for generalized condensers}

5.1. An unconstrained weighted minimum $\alpha$-Riesz energy problem. Consider a generalized condenser $\mathbf{A}=\left(A_{i}\right)_{i \in I}$ in $\mathbb{R}^{n}$ with $p:=$ Card $I \geqslant 2$ such that $I^{+}:=$ $\{1, \ldots, p-1\}$ and $I^{-}:=\{p\}$ (see Section 3.1). Also require that the negative plate $A_{p}$ is closed in $\mathbb{R}^{n}$, while all the positive plates $A_{j}, j \in I^{+}$, are relatively closed subsets of the (open) set $D:=A_{p}^{c}=\mathbb{R}^{n} \backslash A_{p} .{ }^{12}$ For the sake of simplicity, in all that follows assume that $D$ is a domain.

Recall that, by convention (3.1), $c_{\alpha}\left(A_{i}\right)>0$ for all $i \in I$.

When speaking of an external field $\mathbf{f}=\left(f_{i}\right)_{i \in I}$ acting on the vector measures of the class $\mathscr{E}_{\alpha}^{+}\left(\mathbf{A} ; \mathbb{R}^{n}\right)$, we shall always tacitly assume that either Case I or Case II holds, where:

\footnotetext{
${ }^{11}$ If the measure in question is positive, then Lemma 4.3 can be generalized to any bounded $\mu \in \mathfrak{M}^{+}(D)$ such that the Euclidean distance between $S_{D}^{\mu}$ and $\partial D$ is $>0$, see [16, Lemma 3.4].

${ }^{12}$ By [2, Chapter I, Section 3, Proposition 5], this is in agreement with our general requirement that the sets $A_{i}, i \in I$, be locally closed in $\mathbb{R}^{n}$ (see the beginning of Section 3.1).
} 
I. $f_{i} \in \Psi\left(\mathbb{R}^{n}\right)$ for every $i \in I$, and moreover

$$
f_{p}=0 \quad \text { n.e. on } A_{p} \text {. }
$$

II. $f_{i}=s_{i} \kappa_{\alpha}\left(\cdot, \zeta-\zeta^{\prime}\right)$ for every $i \in I$, where $\zeta$ is an extendible (signed) measure on $D$ with $\kappa_{\alpha}(\zeta, \zeta)<\infty$.

Observe that (5.1) holds also in Case II (see (4.2)). Since a set with $c_{\alpha}(\cdot)=0$ carries no (nonzero) measure with finite $\alpha$-Riesz energy [11, Lemma 2.3.1], we thus see that under the stated assumptions no external field acts on the measures from $\mathscr{E}_{\alpha}^{+}\left(A_{p} ; \mathbb{R}^{n}\right)$. Furthermore, $D^{c}$ is $v$-negligible for any $v \in \mathfrak{M i}^{+}\left(A_{j} ; \mathbb{R}^{n}\right), \quad j \in I^{+}$(see Section 2). We are thus led to the following conclusion.

Lemma 5.1. The f-weighted $\alpha$-Riesz energy $G_{\alpha, \mathbf{f}}(\boldsymbol{\mu})$ of $\boldsymbol{\mu} \in \mathscr{E}_{\alpha}^{+}\left(\mathbf{A} ; \mathbb{R}^{n}\right)$ can (equivalently) be defined by the formula ${ }^{13}$

$$
G_{\alpha, \mathbf{f}}(\boldsymbol{\mu})=\kappa_{\alpha}(\boldsymbol{\mu}, \boldsymbol{\mu})+2\langle\mathbf{f}, \boldsymbol{\mu}\rangle=\kappa_{\alpha}(\boldsymbol{\mu}, \boldsymbol{\mu})+2\left\langle\mathbf{f}^{+}, \boldsymbol{\mu}^{+}\right\rangle,
$$

where $\mathbf{f}^{+}:=\left(\left.f_{j}\right|_{D}\right)_{j \in I^{+}}$and $\boldsymbol{\mu}^{+}:=\left(\mu^{j}\right)_{j \in I^{+}}$.

If Case II holds, then for every $\boldsymbol{\mu} \in \mathscr{E}_{\alpha}^{+}\left(\mathbf{A} ; \mathbb{R}^{n}\right)$ we get from (3.4) and (3.8)

$$
G_{\alpha, \mathbf{f}}(\boldsymbol{\mu})=\|R \boldsymbol{\mu}\|_{\alpha}^{2}+2 \sum_{i \in I} s_{i} \kappa_{\alpha}\left(\zeta-\zeta^{\prime}, \mu^{i}\right)=\|R \boldsymbol{\mu}\|_{\alpha}^{2}+2 \kappa_{\alpha}\left(\zeta-\zeta^{\prime}, R \boldsymbol{\mu}\right),
$$

and hence

$$
\infty>G_{\alpha, \mathbf{f}}(\boldsymbol{\mu})=\left\|R \boldsymbol{\mu}+\zeta-\zeta^{\prime}\right\|_{\alpha}^{2}-\left\|\zeta-\zeta^{\prime}\right\|_{\alpha}^{2} \geqslant-\left\|\zeta-\zeta^{\prime}\right\|_{\alpha}^{2}>-\infty .
$$

Thus, in either Case I or Case II

$$
G_{\alpha, \mathbf{f}}(\boldsymbol{\mu}) \geqslant-M>-\infty \quad \text { for all } \boldsymbol{\mu} \in \mathscr{E}_{\alpha}^{+}\left(\mathbf{A} ; \mathbb{R}^{n}\right),
$$

which is clear from (3.8) and (5.2) if Case I holds, or from (5.3) otherwise.

Fix a numerical vector $\mathbf{a}=\left(a_{i}\right)_{i \in I}$ with $a_{i}>0, i \in I$. Using the notations of Section 3.4 with $X=\mathbb{R}^{n}$ and $\kappa=\kappa_{\alpha}$, we obtain from the preceding display

$$
G_{\alpha, \mathbf{f}}\left(\mathbf{A}, \mathbf{a} ; \mathbb{R}^{n}\right):=\inf _{\boldsymbol{\mu} \in \mathscr{E}_{\alpha, \mathbf{f}}^{+}\left(\mathbf{A}, \mathbf{a} ; \mathbb{R}^{n}\right)} G_{\alpha, \mathbf{f}}(\boldsymbol{\mu})>-\infty .
$$

If $\mathscr{E}_{\alpha, \mathbf{f}}^{+}\left(\mathbf{A}, \mathbf{a} ; \mathbb{R}^{n}\right)$ is nonempty, or equivalently if $G_{\alpha, \mathbf{f}}\left(\mathbf{A}, \mathbf{a} ; \mathbb{R}^{n}\right)<\infty$, then we can consider Problem 3.1 on the existence of $\lambda_{\mathbf{A}} \in \mathscr{E}_{\alpha, \mathbf{f}}^{+}\left(\mathbf{A}, \mathbf{a} ; \mathbb{R}^{n}\right)$ with

$$
G_{\alpha, \mathbf{f}}\left(\lambda_{\mathbf{A}}\right)=G_{\alpha, \mathbf{f}}\left(\mathbf{A}, \mathbf{a} ; \mathbb{R}^{n}\right) .
$$

The following theorem shows that, in general, this problem has no solution.

\footnotetext{
${ }^{13}$ Cf. (3.13) with $X=\mathbb{R}^{n}$ and $\kappa=\kappa_{\alpha}$.
} 
Theorem 5.1. Let $D^{c}$ be not $\alpha$-thin at infinity, $I^{+}=\{1\}, c_{g_{D}^{\alpha}}\left(A_{1}\right)=\infty$, and let $\mathbf{a}=\mathbf{1}$ and $\mathbf{f}=\mathbf{0}$, where $\mathbf{1}:=(1,1)$. Then

$$
G_{\alpha, \mathbf{f}}\left(\mathbf{A}, \mathbf{a} ; \mathbb{R}^{n}\right)=\left[c_{g_{D}^{\alpha}}\left(A_{1}\right)\right]^{-1}=0 ;
$$

hence $G_{\alpha, \mathbf{f}}\left(\mathbf{A}, \mathbf{a} ; \mathbb{R}^{n}\right)$ cannot be an actual minimum because $\mathbf{0} \notin \mathscr{E}_{\alpha, \mathbf{f}}^{+}\left(\mathbf{A}, \mathbf{a} ; \mathbb{R}^{n}\right)$.

Proof. Since $G_{\alpha, \mathbf{f}}(\boldsymbol{\mu})=\kappa_{\alpha}(\boldsymbol{\mu}, \boldsymbol{\mu})$ because of $\mathbf{f}=\mathbf{0}$, Problem 3.1 reduces to the problem of minimizing $\kappa_{\alpha}(\boldsymbol{\mu}, \boldsymbol{\mu})$ over $\mathscr{E}_{\alpha}^{+}\left(\mathbf{A}, \mathbf{a} ; \mathbb{R}^{n}\right)$. Thus by (3.8)

$$
G_{\alpha, \mathbf{f}}\left(\mathbf{A}, \mathbf{a} ; \mathbb{R}^{n}\right) \geqslant 0 .
$$

Consider compact sets $K_{\ell} \subset A_{1}, \ell \in \mathbb{N}$, such that $K_{\ell} \uparrow A_{1}$ as $\ell \rightarrow \infty$. By (2.3),

$$
c_{g}\left(K_{\ell}\right) \uparrow c_{g}\left(A_{1}\right)=\infty \quad \text { as } \ell \rightarrow \infty,
$$

and hence there is no loss of generality in assuming that $c_{g}\left(K_{\ell}\right)>0$ for every $\ell \in \mathbb{N}$. Furthermore, since the $\alpha$-Green kernel $g$ is strictly positive definite and moreover perfect (Remark 2.1), we see from (2.2) that $c_{g}\left(K_{\ell}\right)<\infty$ and, by Remark 2.3 , there exists a (unique) $g$-capacitary measure $\lambda_{\ell}$ on $K_{\ell}$, i.e. $\lambda_{\ell} \in \mathscr{E}_{g}^{+}\left(K_{\ell}, 1 ; D\right)$ with

$$
\left\|\lambda_{\ell}\right\|_{g}^{2}=1 / c_{g}\left(K_{\ell}\right)<\infty .
$$

According to Lemma 4.3 with $\lambda_{\ell}$ in place of $\mu, \kappa_{\alpha}\left(\lambda_{\ell}, \lambda_{\ell}\right)$ is finite along with $g\left(\lambda_{\ell}, \lambda_{\ell}\right)$. Hence, by Lemma 4.2 ,

$$
\left\|\lambda_{\ell}\right\|_{g}^{2}=\left\|\lambda_{\ell}-\lambda_{\ell}^{\prime}\right\|_{\alpha}^{2}
$$

Applying Theorem 4.1, we get $\boldsymbol{\mu}_{\ell}:=\left(\lambda_{\ell}, \lambda_{\ell}^{\prime}\right) \in \mathscr{E}_{\alpha, \mathbf{f}}^{+}\left(\mathbf{A}, \mathbf{a} ; \mathbb{R}^{n}\right)$, which together with the two preceding displays and (3.8) and (5.5) gives

$$
1 / c_{g}\left(K_{\ell}\right)=\left\|\lambda_{\ell}-\lambda_{\ell}^{\prime}\right\|_{\alpha}^{2}=\kappa_{\alpha}\left(\boldsymbol{\mu}_{\ell}, \boldsymbol{\mu}_{\ell}\right) \geqslant G_{\alpha, \mathbf{f}}\left(\mathbf{A}, \mathbf{a} ; \mathbb{R}^{n}\right) \geqslant 0 .
$$

Letting here $\ell \rightarrow \infty$, we obtain the theorem from (5.6).

Using the electrostatic interpretation, which is possible for the Coulomb kernel $|x-y|^{-1}$ on $\mathbb{R}^{3}$, we say that under the hypotheses of Theorem 5.1 a short-circuit occurs between the oppositely signed plates of the generalized condenser A. It is therefore meaningful to ask what kinds of additional requirements on the objects in question will prevent this blow-up effect, and secure that a solution to the corresponding minimum $\alpha$-Riesz energy problem does exist. To this end we have succeeded in working out a substantive theory by imposing a proper vector constraint on the vector measures under consideration. 
5.2. A constrained weighted minimum $\alpha$-Riesz energy problem. Let $\mathbf{A}$, a and $\mathbf{f}$ be as at the beginning of Section 5.1. In the rest of the paper we assume additionally that $A_{p}\left(=D^{c}\right)$ is not $\alpha$-thin at infinity and

$$
a_{p}=\sum_{j \in I^{+}} a_{j} .
$$

Using notation of Section 3.5, fix $\xi=\left(\xi^{i}\right)_{i \in I}$ with

$$
\xi^{j} \in \mathbb{C}\left(A_{j} ; \mathbb{R}^{n}\right) \cap \mathscr{E}_{\alpha}^{+}\left(A_{j} ; \mathbb{R}^{n}\right) \quad \text { for all } j \in I^{+} \text {, and } \xi^{p}=\infty \text {. }
$$

Unless explicitly stated otherwise, for these $\mathbf{A}, \mathbf{a}, \mathbf{f}$, and $\boldsymbol{\xi}$ we shall always require that

$$
G_{\alpha, \mathbf{f}}^{\xi}\left(\mathbf{A}, \mathbf{a} ; \mathbb{R}^{n}\right)<\infty .
$$

The main purpose of this paper is to analyze Problem 3.2 on the existence of $\lambda_{\mathbf{A}}^{\xi} \in \mathscr{E}_{\alpha, \mathbf{f}}^{\xi}\left(\mathbf{A}, \mathbf{a} ; \mathbb{R}^{n}\right)$ with $G_{\alpha, \mathbf{f}}\left(\lambda_{\mathbf{A}}^{\xi}\right)=G_{\alpha, \mathbf{f}}^{\xi}\left(\mathbf{A}, \mathbf{a} ; \mathbb{R}^{n}\right)$. Recall that $\mathfrak{G}_{\alpha, \mathbf{f}}^{\xi}\left(\mathbf{A}, \mathbf{a} ; \mathbb{R}^{n}\right)$ denotes the class of all solutions to this problem (provided these exist). According to Lemma 3.6, any two solutions are $R$-equivalent; hence, by Corollary 3.1, $\mathfrak{\Xi}_{\alpha, \mathbf{f}}^{\xi}\left(\mathbf{A}, \mathbf{a} ; \mathbb{R}^{n}\right)$ reduces to a single element whenever all the $A_{j}$, $j \in I^{+}$, are mutually essentially disjoint, i.e. with $c_{\alpha}\left(A_{i} \cap A_{j}\right)=0$ for all $i, j \in I^{+}$, $i \neq j$.

Conditions on $\mathbf{A}, \mathbf{f}$ and $\boldsymbol{\xi}$ which guarantee that (5.9) holds are given in the following Lemma 5.2. Write

$$
A_{j}^{\circ}:=\left\{x \in A_{j}:\left|f_{j}(x)\right|<\infty\right\}, \quad j \in I^{+} .
$$

Leмma 5.2. The (permanent) requirement (5.9) holds if either Case II takes place, or (in the presence of Case I) if

$$
\xi^{j}\left(A_{j}^{\circ}\right)>a_{j} \quad \text { for all } j \in I^{+} .
$$

Proof. Assume first that (5.11) holds. Then there is for every $j \in I^{+}$a compact set $K_{j} \subset A_{j}^{\circ}$ such that $\xi^{j}\left(K_{j}\right)>a_{j}$ and $\left|f_{j}\right| \leqslant M_{j}<\infty$ on $K_{j}$ for some constant $M_{j}$ (see (5.10)). Define $\boldsymbol{\mu}:=\left(\mu^{i}\right)_{i \in I}$, where $\mu^{j}:=\left.a_{j} \xi^{j}\right|_{K_{j}} / \xi^{j}\left(K_{j}\right)$ for all $j \in I^{+}$and $\mu^{p}$ is any measure from $\mathscr{E}_{\alpha}^{+}\left(A_{p}, a_{p} ; \mathbb{R}^{n}\right.$ ) (such $\mu^{p}$ exists since $\left.c_{\alpha}\left(A_{p}\right)>0\right)$. Noting that $\left.\xi^{j}\right|_{K_{j}} \in \mathscr{E}_{\alpha}^{+}\left(K_{j} ; \mathbb{R}^{n}\right)$ for all $j \in I^{+}$by (5.8), we get $\boldsymbol{\mu} \in \mathscr{E}_{\alpha, \mathbf{f}}^{\xi}\left(\mathbf{A}, \mathbf{a} ; \mathbb{R}^{n}\right)$, which yields (5.9). To complete the proof, it is left to observe that (5.11) holds automatically if Case II takes place, because then $\kappa_{\alpha}\left(\cdot, \zeta-\zeta^{\prime}\right)$ is finite n.e. on $\mathbb{R}^{n}$, hence $\xi^{j}$-a.e. for all $j \in I^{+}$by $[11$, Lemma 2.3.1].

The theory developed in the present study includes sufficient and/or necessary conditions for the existence of solutions $\lambda_{\mathbf{A}}^{\xi}=\left(\lambda_{\mathbf{A}}^{i}\right)_{i \in I}$ to Problem 3.2 with $\mathbf{A}$, a, $\mathbf{f}$ and $\boldsymbol{\xi}$ chosen above (see Theorems 7.1 and 7.2). We also provide 
descriptions of the f-weighted $\alpha$-Riesz vector potentials of the solutions $\lambda_{\mathbf{A}}^{\xi}$, single out their characteristic properties, and analyze the supports of the $\lambda_{\mathrm{A}}^{i}$, $i \in I$ (see Theorems 7.3, 7.4 and 7.5). These results are illustrated in Examples 10.1 and 10.2. See also Section 7.2 for an extension of the theory to the case where $\xi^{p} \neq \infty$. The proofs of Theorems 7.1-7.5 are given in Sections 8 and 9; they are substantially based on Theorem 6.1 which is a subject of the next section.

REMARK 5.1. Under the (permanent) assumption (5.9), $G_{\alpha, \mathbf{f}}^{\xi}\left(\mathbf{A}, \mathbf{a} ; \mathbb{R}^{n}\right)$ is actually finite, because $G_{\alpha, \mathbf{f}}^{\xi}\left(\mathbf{A}, \mathbf{a} ; \mathbb{R}^{n}\right)>-\infty$ follows from (5.4) in view of the inclusion $\mathscr{E}_{\alpha, \mathbf{f}}^{\xi}\left(\mathbf{A}, \mathbf{a} ; \mathbb{R}^{n}\right) \subset \mathscr{E}_{\alpha, \mathbf{f}}^{+}\left(\mathbf{A}, \mathbf{a} ; \mathbb{R}^{n}\right)$.

\section{Relations between constrained minimum $\alpha$-Riesz and $\alpha$-Green energy problems}

Throughout this section, $\mathbf{A}, \mathbf{a}, \mathbf{f}$ and $\boldsymbol{\xi}$ are as indicated at the beginning of Section 5.2, except for (5.9) which is temporarily not required. The aim of Theorem 6.1 below is to establish a relationship between, on the one hand, the solvability (or the non-solvability) of Problem 3.2 for $\mathbb{R}^{n}, \kappa_{\alpha}, \mathbf{A}, \mathbf{a}, \mathbf{f}, \boldsymbol{\xi}$ and, on the other hand, that for $D, g=g_{D}^{\alpha}, \mathbf{A}^{+}, \mathbf{a}^{+}, \mathbf{f}^{+}$and $\boldsymbol{\xi}^{+}$, where

$$
\mathbf{A}^{+}:=\left(A_{j}\right)_{j \in I^{+}}, \quad \mathbf{a}^{+}:=\left(a_{j}\right)_{j \in I^{+}}, \quad \mathbf{f}^{+}:=\left(\left.f_{j}\right|_{D}\right)_{j \in I^{+}}, \quad \boldsymbol{\xi}^{+}:=\left(\xi^{j}\right)_{j \in I^{+}} .
$$

(Note that $\mathbf{A}^{+}$is a standard condenser in $X=D$ consisting of only positive plates.) Observe that since for every given $j \in I^{+}$we have

$$
\mathfrak{M}^{+}\left(A_{j} ; \mathbb{R}^{n}\right) \subset \mathfrak{M}^{+}\left(A_{j} ; D\right),
$$

the measure $\xi^{j}$ can certainly be thought of as an element of $\mathfrak{C}\left(A_{j} ; D\right)$.

For any $\boldsymbol{\mu}=\left(\mu^{i}\right)_{i \in I} \in \mathfrak{M}^{+}\left(\mathbf{A} ; \mathbb{R}^{n}\right)$ write $\boldsymbol{\mu}^{+}:=\left(\mu^{j}\right)_{j \in I^{+}}$; then $\boldsymbol{\mu}^{+}$belongs to $\mathfrak{M}^{+}\left(\mathbf{A}^{+} ; D\right)$ by $(6.1)$. If moreover $\kappa_{\alpha}(\boldsymbol{\mu}, \boldsymbol{\mu})<\infty$, then $\boldsymbol{\mu}^{+}$belongs to $\mathscr{E}_{\alpha}^{+}\left(\mathbf{A}^{+} ; \mathbb{R}^{n}\right) ;$ and also to $\mathscr{E}_{g}^{+}\left(\mathbf{A}^{+} ; D\right)$ which is clear from $(4.7)$.

THEOREM 6.1. Under the just mentioned assumptions on $\mathbf{A}, \mathbf{a}, \mathbf{f}$ and $\boldsymbol{\xi}$,

$$
G_{\alpha, \mathbf{f}}^{\xi}\left(\mathbf{A}, \mathbf{a} ; \mathbb{R}^{n}\right)=G_{g, \mathbf{f}^{+}}^{\xi^{+}}\left(\mathbf{A}^{+}, \mathbf{a}^{+} ; D\right) .
$$

If moreover these (equal) extremal values are finite, then the class $\boldsymbol{\Xi}_{\alpha, \mathbf{f}}^{\xi}\left(\mathbf{A}, \mathbf{a} ; \mathbb{R}^{n}\right)$ is nonempty if and only if so is $\mathfrak{S}_{g, \mathbf{f}^{+}}^{\xi^{+}}\left(\mathbf{A}^{+}, \mathbf{a}^{+} ; D\right)$, and in the affirmative case the following two assertions are equivalent for any $\lambda_{\mathbf{A}}=\left(\lambda_{\mathbf{A}}^{i}\right)_{i \in I} \in \mathfrak{M}^{+}\left(\mathbf{A} ; \mathbb{R}^{n}\right)$ :

(i) $\lambda_{\mathbf{A}} \in \boldsymbol{\Xi}_{\alpha, \mathbf{f}}^{\xi}\left(\mathbf{A}, \mathbf{a} ; \mathbb{R}^{n}\right)$.

(ii) $\lambda_{\mathbf{A}}^{+}=\left(\lambda_{\mathbf{A}}^{j}\right)_{j \in I^{+}} \in \mathfrak{S}_{g, \mathbf{f}^{+}}^{\xi^{+}}\left(\mathbf{A}^{+}, \mathbf{a}^{+} ; D\right)$ and, in addition,

$$
\lambda_{\mathbf{A}}^{p}=\left(\sum_{j \in I^{+}} \lambda_{\mathbf{A}}^{j}\right)^{\prime} .
$$


Proof. We begin by establishing the inequality

$$
G_{g, \mathbf{f}^{+}}^{\xi^{+}}\left(\mathbf{A}^{+}, \mathbf{a}^{+} ; D\right) \geqslant G_{\alpha, \mathbf{f}}^{\xi}\left(\mathbf{A}, \mathbf{a} ; \mathbb{R}^{n}\right) .
$$

Assuming $G_{g, \mathbf{f}^{+}}^{\xi^{+}}\left(\mathbf{A}^{+}, \mathbf{a}^{+} ; D\right)<\infty$, choose $\boldsymbol{\mu}=\left(\mu^{j}\right)_{j \in I^{+}} \in \mathscr{E}_{g, \mathbf{f}^{+}}^{\xi^{+}}\left(\mathbf{A}^{+}, \mathbf{a}^{+} ; D\right)$. According to (3.8) and (3.13) with $X=D$ and $\kappa=g$, then

$$
G_{g, \mathbf{f}^{+}}(\boldsymbol{\mu})=g(\boldsymbol{\mu}, \boldsymbol{\mu})+2\left\langle\mathbf{f}^{+}, \boldsymbol{\mu}\right\rangle=\left\|R_{\mathbf{A}^{+}} \boldsymbol{\mu}\right\|_{g}^{2}+2\left\langle\mathbf{f}^{+}, \boldsymbol{\mu}\right\rangle .
$$

Being bounded, each of the $\mu^{j}, j \in I^{+}$, is extendible (see Section 4). Furthermore, the extension in question has finite $\alpha$-Riesz energy, for so does the extension of the constraint $\xi^{j}$ by (5.8). Applying (4.9) to $R_{\mathbf{A}^{+}} \boldsymbol{\mu} \in \mathscr{E}_{\alpha}^{+}\left(A^{+} ; \mathbb{R}^{n}\right)$ in place of $\mu$, we thus get

$$
G_{g, \mathbf{f}^{+}}(\boldsymbol{\mu})=\left\|R_{\mathbf{A}^{+}} \boldsymbol{\mu}-\left(R_{\mathbf{A}^{+}} \boldsymbol{\mu}\right)^{\prime}\right\|_{\alpha}^{2}+2\left\langle\mathbf{f}^{+}, \boldsymbol{\mu}\right\rangle .
$$

Since $A_{p}\left(=D^{c}\right)$ is not $\alpha$-thin at infinity, we conclude from (4.5) and (5.7) that $\left(R_{\mathbf{A}^{+}} \boldsymbol{\mu}\right)^{\prime} \in \mathscr{E}_{\alpha}^{+}\left(A_{p}, a_{p} ; \mathbb{R}^{n}\right)$, and therefore $\tilde{\boldsymbol{\mu}}=\left(\tilde{\mu}^{i}\right)_{i \in I} \in \mathscr{E}_{\alpha}^{\xi}\left(\mathbf{A}, \mathbf{a} ; \mathbb{R}^{n}\right)$ where

$$
\tilde{\boldsymbol{\mu}}^{+}=\boldsymbol{\mu} \quad \text { and } \quad \tilde{\mu}^{p}=\left(R_{\mathbf{A}^{+}} \boldsymbol{\mu}\right)^{\prime}=\left(\sum_{j \in I^{+}} \mu^{j}\right)^{\prime} .
$$

Here we have used the (permanent) assumption that $\xi^{p}=\infty$. Furthermore,

$$
\langle\mathbf{f}, \tilde{\boldsymbol{\mu}}\rangle=\left\langle\mathbf{f}^{+}, \boldsymbol{\mu}\right\rangle\langle\infty,
$$

the equality being valid because $f^{p}=0$ n.e. on $A_{p}$ (see Section 5.1), hence $\tilde{\mu}^{p}$-a.e. by [11, Lemma 2.3.1], and also because $D^{c}$ is $\mu^{j}$-negligible for every $j \in I^{+}$. Thus actually $\tilde{\boldsymbol{\mu}} \in \mathscr{E}_{\alpha, \mathbf{f}}^{\xi}\left(\mathbf{A}, \mathbf{a} ; \mathbb{R}^{n}\right)$; and moreover $G_{\alpha, \mathbf{f}}(\tilde{\boldsymbol{\mu}})=G_{g, \mathbf{f}^{+}}(\boldsymbol{\mu})$, the latter being clear from the three preceding displays. This yields

$$
G_{g, \mathbf{f}^{+}}(\boldsymbol{\mu})=G_{\alpha, \mathbf{f}}(\tilde{\boldsymbol{\mu}}) \geqslant G_{\alpha, \mathbf{f}}^{\xi}\left(\mathbf{A}, \mathbf{a} ; \mathbb{R}^{n}\right) .
$$

Letting here $\boldsymbol{\mu}$ range over $\mathscr{E}_{g, \mathbf{f}^{+}}^{\xi^{+}}\left(\mathbf{A}^{+}, \mathbf{a}^{+} ; D\right)$ results in (6.4).

On the other hand, in view of (4.7) and (5.2) for any $\boldsymbol{v} \in \mathscr{E}_{\alpha, \mathbf{f}}^{\boldsymbol{\xi}}\left(\mathbf{A}, \mathbf{a} ; \mathbb{R}^{n}\right)$ we have $\boldsymbol{v}^{+} \in \mathscr{E}_{g, \mathbf{f}^{+}}^{\xi^{+}}\left(\mathbf{A}^{+}, \mathbf{a}^{+} ; D\right)$. Thus, by (3.8), (4.4), (4.9) and (5.2),

$$
\begin{aligned}
G_{\alpha, \mathbf{f}}(\boldsymbol{v}) & =\kappa_{\alpha}(\boldsymbol{v}, \boldsymbol{v})+2\left\langle\mathbf{f}^{+}, \boldsymbol{v}^{+}\right\rangle=\left\|R_{\mathbf{A}} \boldsymbol{v}^{+}-v^{p}\right\|_{\alpha}^{2}+2\left\langle\mathbf{f}^{+}, \boldsymbol{v}^{+}\right\rangle \\
& \geqslant\left\|R_{\mathbf{A}} \boldsymbol{v}^{+}-\left(R_{\mathbf{A}} \boldsymbol{v}^{+}\right)^{\prime}\right\|_{\alpha}^{2}+2\left\langle\mathbf{f}^{+}, \boldsymbol{v}^{+}\right\rangle=\left\|R_{\mathbf{A}} \boldsymbol{v}^{+}\right\|_{g}^{2}+2\left\langle\mathbf{f}^{+}, \boldsymbol{v}^{+}\right\rangle \\
& =g\left(\boldsymbol{v}^{+}, \boldsymbol{v}^{+}\right)+2\left\langle\mathbf{f}^{+}, \boldsymbol{v}^{+}\right\rangle=G_{g, \mathbf{f}^{+}}\left(\boldsymbol{v}^{+}\right) \geqslant G_{g, \mathbf{f}^{+}}^{\xi^{+}}\left(\mathbf{A}^{+}, \mathbf{a}^{+} ; D\right) .
\end{aligned}
$$

Letting here $\boldsymbol{v}$ vary over $\mathscr{E}_{\alpha, \mathbf{f}}^{\xi}\left(\mathbf{A}, \mathbf{a} ; \mathbb{R}^{n}\right)$ and then combining the inequality thus obtained with (6.4), we arrive at (6.2).

Now suppose that there exists $\boldsymbol{\mu}=\left(\mu^{j}\right)_{j \in I^{+}} \in \mathbb{S}_{g, \mathbf{f}^{+}}^{\xi^{+}}\left(\mathbf{A}^{+}, \mathbf{a}^{+} ; D\right)$. Define $\tilde{\boldsymbol{\mu}}=\left(\tilde{\mu}^{i}\right)_{i \in I}$ as in (6.5). Then the same arguments as those applied in the first 
paragraph of this proof enable us to see that $\tilde{\boldsymbol{\mu}} \in \mathscr{E}_{\alpha, \mathbf{f}}^{\xi}\left(\mathbf{A}, \mathbf{a} ; \mathbb{R}^{n}\right)$ and also that $G_{\alpha, \mathbf{f}}(\tilde{\boldsymbol{\mu}})=G_{g, \mathbf{f}^{+}}(\boldsymbol{\mu})$. The latter yields

$$
G_{\alpha, \mathbf{f}}(\tilde{\boldsymbol{\mu}})=G_{g, \mathbf{f}^{+}}^{\xi^{+}}\left(\mathbf{A}^{+}, \mathbf{a}^{+} ; D\right) .
$$

Substituting (6.2) into the last display shows that, actually, $\tilde{\boldsymbol{\mu}} \in \boldsymbol{\Xi}_{\alpha, \mathbf{f}}^{\xi}\left(\mathbf{A}, \mathbf{a} ; \mathbb{R}^{n}\right)$, which in view of the latter relation in (6.5) proves that, indeed, (ii) implies (i).

As for the converse implication, let there be $\boldsymbol{v}=\left(v^{i}\right)_{i \in I} \in \boldsymbol{\Xi}_{\alpha, \mathbf{f}}^{\xi}\left(\mathbf{A}, \mathbf{a} ; \mathbb{R}^{n}\right)$. Then $\boldsymbol{v}^{+} \in \mathscr{E}_{g, \mathbf{f}^{+}}^{\xi^{+}}\left(\mathbf{A}^{+}, \mathbf{a}^{+} ; D\right)$ (see the second paragraph of the proof) and (6.6) holds. Since for this $\boldsymbol{v}$ the first term in (6.6) equals $G_{\alpha, \mathbf{f}}^{\xi}\left(\mathbf{A}, \mathbf{a} ; \mathbb{R}^{n}\right)$, we see from (6.2) that equality prevails in either of the two inequalities in (6.6). Thus, $\boldsymbol{v}^{+} \in \mathfrak{S}_{g, \mathbf{f}^{+}}^{\xi^{+}}\left(\mathbf{A}^{+}, \mathbf{a}^{+} ; D\right)$ and also $v^{p}=\left(R \boldsymbol{v}^{+}\right)^{\prime}$, the latter being clear from (4.4).

\section{Main results}

Throughout Section 7 we keep all the assumptions on $\mathbf{A}$, a, f and $\boldsymbol{\xi}$ imposed at the beginning of Section 5.2, except for (5.9). ${ }^{14}$

\subsection{Formulations of the main results.}

THEOREM 7.1. Suppose moreover that (5.9) is fulfilled and also

$$
\xi^{j}\left(A_{j}\right)<\infty \quad \text { for all } j \in I^{+} .
$$

Then the class $\boldsymbol{S}_{\alpha, \mathbf{f}}^{\xi}\left(\mathbf{A}, \mathbf{a} ; \mathbb{R}^{n}\right)$ of all solutions to Problem 3.2 is nonempty, and for any one of its elements $\lambda_{\mathbf{A}}^{\xi}=\left(\lambda_{\mathbf{A}}^{i}\right)_{i \in I}$ we have $\lambda_{\mathbf{A}}^{p}=\left(\sum_{j \in I^{+}} \lambda_{\mathbf{A}}^{j}\right)^{\prime}$.

Theorem 7.1 is sharp in the sense that it no longer holds if (7.1) is omitted from its hypotheses (see the following Theorem 7.2).

THEOREM 7.2. Condition (7.1) is in general also necessary for the solvability of Problem 3.2. More precisely, suppose that $I^{+}=\{1\}, c_{\alpha}\left(A_{1}\right)=\infty$ and that Case II holds with $\zeta \geqslant 0$. Then there is a constraint $\xi^{1} \in \mathbb{C}\left(A_{1} ; \mathbb{R}^{n}\right) \cap$ $\mathscr{E}_{\alpha}^{+}\left(A_{1} ; \mathbb{R}^{n}\right)$ with $\xi^{1}\left(A_{1}\right)=\infty$ such that the corresponding Problem 3.2 is unsolvable.

The following three assertions provide descriptions of the f-weighted $\alpha$-Riesz potentials $W_{\alpha, \mathbf{f}}^{\lambda_{\mathbf{A}}^{\xi}}$, cf. (3.12), of the solutions $\lambda_{\mathbf{A}}^{\xi}=\left(\lambda_{\mathbf{A}}^{i}\right)_{i \in I} \in \mathfrak{G}_{\alpha, \mathbf{f}}^{\xi}\left(\mathbf{A}, \mathbf{a} ; \mathbb{R}^{n}\right)$ (whenever these exist), single out their characteristic properties, and analyze the supports of the $\lambda_{\mathrm{A}}^{i}, i \in I$.

\footnotetext{
${ }^{14}$ Under the hypotheses of any of Theorems 7.2-7.5, (5.9) holds in consequence of Lemma 5.2.
} 
THEOREM 7.3. Let (5.11) hold, and let each $f_{j}, j \in I^{+}$, be lower bounded on $A_{j}$. Fix $\lambda_{\mathbf{A}} \in \mathscr{E}_{\alpha, \mathbf{f}}^{\xi}\left(\mathbf{A}, \mathbf{a} ; \mathbb{R}^{n}\right)$ (which exists). Then the following two assertions are equivalent:

(i) $\lambda_{\mathbf{A}} \in \mathfrak{S}_{\alpha, \mathbf{f}}^{\xi}\left(\mathbf{A}, \mathbf{a} ; \mathbb{R}^{n}\right)$.

(ii) There exists a vector $\left(c_{j}\right)_{j \in I^{+}} \in \mathbb{R}^{p-1}$ such that for all $j \in I^{+}$

$$
\begin{aligned}
& W_{\alpha, \mathbf{f}}^{\lambda_{\mathbf{A}}, j} \geqslant c_{j} \quad\left(\xi^{j}-\lambda_{\mathbf{A}}^{j}\right) \text {-a.e. }, \\
& W_{\alpha, \mathbf{f}}^{\lambda_{\mathbf{A}}, j} \leqslant c_{j} \quad \lambda_{\mathbf{A}}^{j} \text {-a.e. },
\end{aligned}
$$

and in addition we have

$$
W_{\alpha, \mathbf{f}}^{\lambda_{\mathbf{A}}, p}=0 \quad \text { n.e. on } A_{p} .
$$

If moreover Case II takes place, then relation (7.4) actually holds for every $W_{\alpha, \mathbf{f}}^{\lambda_{\mathrm{A}}, i}, i \in I$, and it takes now the form

$$
W_{\alpha, \mathbf{f}}^{\lambda_{\mathbf{A}}, i}=0 \quad \text { on } A_{p} \backslash I_{\alpha, A_{p}}, \quad i \in I,
$$

where $I_{\alpha, A_{p}}$ denotes the set of all $\alpha$-irregular (boundary) points of $A_{p}$.

REMARK 7.1. The lower boundedness of $f_{j}, j \in I^{+}$, required in Theorem 7.3, holds automatically provided that Case I takes place. Furthermore, in Case I relation (7.3) is equivalent to the following apparently stronger assertion:

$$
W_{\alpha, \mathbf{f}}^{\lambda_{\mathbf{A}}, j} \leqslant c_{j} \quad \text { on } S_{D}^{\lambda_{\mathrm{A}}^{j}} .
$$

Let $\breve{Q}$ denote the $\kappa_{\alpha}$-reduced kernel of $Q \subset \mathbb{R}^{n}[19$, p. 164], which is the set of all $x \in Q$ such that $c_{\alpha}(B(x, r) \cap Q)>0$ for any $r>0$.

For the sake of simplicity of formulation, in the following Theorem 7.4 we assume that in the case $\alpha=2$ the domain $D$ is simply connected.

THEOREM 7.4. If a solution $\lambda_{\mathbf{A}}^{\xi}=\left(\lambda_{\mathbf{A}}^{i}\right)_{i \in I} \in \Xi_{\alpha, \mathbf{f}}^{\xi}\left(\mathbf{A}, \mathbf{a} ; \mathbb{R}^{n}\right)$ exists, then

$$
S_{\mathbb{R}^{n}}^{\lambda^{p}}= \begin{cases}\breve{A_{p}} & \text { if } \alpha<2, \\ \partial D & \text { if } \alpha=2 .\end{cases}
$$

Suppose now that $I^{+}=\{1\}, \mathbf{a}=\mathbf{1}, \mathbf{f}=\mathbf{0}$, and let there exist a solution $\lambda_{\mathbf{A}}^{\xi}=\left(\lambda_{\mathbf{A}}^{1}, \lambda_{\mathbf{A}}^{2}\right) \in \mathfrak{S}_{\alpha, \mathbf{f}}^{\xi}\left(\mathbf{A}, \mathbf{1} ; \mathbb{R}^{n}\right)$. (According to Corollary 3.1, under the stated assumption $I^{+}=\{1\}$ the solution $\lambda_{\mathrm{A}}^{\xi}$ is unique.) Then, equivalently, $\lambda:=$ $R_{\mathbf{A}} \lambda_{\mathbf{A}}^{\xi}=\lambda_{\mathbf{A}}^{1}-\lambda_{\mathbf{A}}^{2}$ is a (unique) solution to the minimum $\alpha$-Riesz energy problem

$$
\inf \kappa_{\alpha}(\mu, \mu) \text {, }
$$

where $\mu$ ranges over all (signed scalar Radon) measures with $\mu^{+} \in \mathscr{E}_{\alpha}^{\xi^{1}}\left(A_{1}, 1\right.$; $\left.\mathbb{R}^{n}\right)$ and $\mu^{-} \in \mathscr{E}_{\alpha}^{+}\left(A_{2}, 1 ; \mathbb{R}^{n}\right)$. Since $\mathbf{f}=\mathbf{0}$, we also see from (3.9) and (3.12) 
that

$$
\kappa_{\alpha}(\cdot, \lambda)=s_{i} \kappa_{\alpha}^{\lambda_{\mathbf{A}}^{\xi}, i}(\cdot)=s_{i} W_{\alpha, \mathbf{f}}^{\lambda_{\mathbf{A}}^{\xi}, i}(\cdot) \quad \text { n.e. on } \mathbb{R}^{n}, \quad i=1,2 .
$$

THEOREM 7.5. With these assumptions and notations, we have

$$
\kappa_{\alpha}(\cdot, \lambda)=\left\{\begin{array}{lll}
g_{D}^{\alpha}\left(\cdot, \lambda^{+}\right) & \text {n.e. on } & D, \\
0 & \text { on } & D^{c} \backslash I_{\alpha, D^{c}} .
\end{array}\right.
$$

Furthermore, assertion (ii) of Theorem 7.3 holds, where (7.2) and (7.3) take now the form

$$
\begin{aligned}
& \kappa_{\alpha}(\cdot, \lambda)=c_{1}\left(\xi^{1}-\lambda^{+}\right) \text {-a.e. }, \\
& \kappa_{\alpha}(\cdot, \lambda) \leqslant c_{1} \quad \text { on } \mathbb{R}^{n}
\end{aligned}
$$

with $0<c_{1}<\infty$. In addition, (7.10) and (7.11) together with $\kappa_{\alpha}(\cdot, \lambda)=0$ n.e. on $D^{c}$ (cf. (7.9)) determine uniquely the solution to the problem (7.7) among the admissible measures $\mu$. If moreover $\kappa_{\alpha}\left(\cdot, \xi^{1}\right)$ is (finitely) continuous on $D$, then also

$$
\begin{gathered}
\kappa_{\alpha}(\cdot, \lambda)=c_{1} \quad \text { on } S_{D}^{\xi^{1}-\lambda^{+}}, \\
c_{g_{D}^{\alpha}}\left(S_{D}^{\xi^{1}-\lambda^{+}}\right)<\infty .
\end{gathered}
$$

Omitting now the requirement of the continuity of $\kappa_{\alpha}\left(\cdot, \xi^{1}\right)$, assume further that $\alpha<2$ and $m_{n}\left(D^{c}\right)>0$. Then

$$
\begin{gathered}
S_{D}^{\lambda^{+}}=S_{D}^{\xi^{1}} \\
\kappa_{\alpha}(\cdot, \lambda)<c_{1} \quad \text { on } D \backslash S_{D}^{\xi^{1}} \quad\left(=D \backslash S_{D}^{\lambda^{+}}\right) .
\end{gathered}
$$

7.2. An extension of the theory. Parallel with Problem 3.2 for a constraint $\xi$ given by (5.8) and acting only on measures carried by the positive plates $A_{j}$, $j \in I^{+}$, of the generalized condenser $\mathbf{A}=\left(A_{i}\right)_{i \in I}$, consider also Problem 3.2 for $\boldsymbol{\sigma}=\left(\sigma^{i}\right)_{i \in I} \in \mathfrak{M}^{+}\left(\mathbf{A} ; \mathbb{R}^{n}\right)$ (in place of $\boldsymbol{\xi}$ ) defined as follows:

$$
\sigma^{j}=\xi^{j} \quad \text { for all } j \in I^{+}, \quad \sigma^{p} \geqslant\left(\sum_{j \in I^{+}} \sigma^{j}\right)^{\prime}\left[=\left(\sum_{j \in I^{+}} \xi^{j}\right)^{\prime}\right] \text {. }
$$

Since in consequence of (4.5), (5.7) and (7.16) we have $\sigma^{p}\left(A_{p}\right)>a_{p}$, the measure $\sigma$ thus defined can be thought of as an element of $\mathfrak{C}\left(\mathbf{A} ; \mathbb{R}^{n}\right)$. In contrast to $\boldsymbol{\xi}$, the constraint $\boldsymbol{\sigma}$ is acting on all the components of $\boldsymbol{\mu} \in \mathscr{E}_{\alpha}^{+}\left(\mathbf{A}, \mathbf{a} ; \mathbb{R}^{n}\right)$. Also note that $\sigma^{p}\left(A_{p}\right)$ and $\kappa_{\alpha}\left(\sigma^{p}, \sigma^{p}\right)$ may both be infinite.

THEOREM 7.6. The following identity holds:

$$
G_{\alpha, \mathbf{f}}^{\boldsymbol{\sigma}}\left(\mathbf{A}, \mathbf{a} ; \mathbb{R}^{n}\right)=G_{\alpha, \mathbf{f}}^{\xi}\left(\mathbf{A}, \mathbf{a} ; \mathbb{R}^{n}\right) .
$$


If moreover these (equal) extremal values are finite, then Problem 3.2 for $\mathbf{A}, \mathbf{a}, \mathbf{f}$ and $\boldsymbol{\xi}$ is solvable if and only if so is that for $\mathbf{A}, \mathbf{a}, \mathbf{f}$ and $\boldsymbol{\sigma}$, and in the affirmative case

$$
\boldsymbol{\Xi}_{\alpha, \mathbf{f}}^{\sigma}\left(\mathbf{A}, \mathbf{a} ; \mathbb{R}^{n}\right)=\boldsymbol{\Xi}_{\alpha, \mathbf{f}}^{\xi}\left(\mathbf{A}, \mathbf{a} ; \mathbb{R}^{n}\right) .
$$

Proof. Indeed, $G_{\alpha, \mathbf{f}}^{\boldsymbol{\sigma}}\left(\mathbf{A}, \mathbf{a} ; \mathbb{R}^{n}\right) \geqslant G_{\alpha, \mathbf{f}}^{\xi}\left(\mathbf{A}, \mathbf{a} ; \mathbb{R}^{n}\right)$ follows from the relation

$$
\mathscr{E}_{\alpha, \mathbf{f}}^{\boldsymbol{\sigma}}\left(\mathbf{A}, \mathbf{a} ; \mathbb{R}^{n}\right) \subset \mathscr{E}_{\alpha, \mathbf{f}}^{\xi}\left(\mathbf{A}, \mathbf{a} ; \mathbb{R}^{n}\right) .
$$

To establish the converse inequality, assume that $G_{\alpha, \mathbf{f}}^{\xi}\left(\mathbf{A}, \mathbf{a} ; \mathbb{R}^{n}\right)<\infty$ and fix $\boldsymbol{v} \in \mathscr{E}_{\alpha, \mathbf{f}}^{\xi}\left(\mathbf{A}, \mathbf{a} ; \mathbb{R}^{n}\right)$. Define $\tilde{\boldsymbol{v}}=\left(\tilde{\boldsymbol{v}}^{i}\right)_{i \in I} \in \mathfrak{M}^{+}\left(\mathbf{A} ; \mathbb{R}^{n}\right)$ by the equalities

$$
\tilde{\boldsymbol{v}}^{+}=\boldsymbol{v}^{+} \quad \text { and } \quad \tilde{v}^{p}=\left(R_{\mathrm{A}} \boldsymbol{v}^{+}\right)^{\prime} .
$$

Clearly $\tilde{\boldsymbol{v}} \in \mathscr{E}_{\alpha}^{+}\left(\mathbf{A} ; \mathbb{R}^{n}\right)$, and moreover $\tilde{\boldsymbol{v}} \in \mathscr{E}_{\alpha}^{+}\left(\mathbf{A}, \mathbf{a} ; \mathbb{R}^{n}\right)$ which follows from (4.5), (5.7) and (7.20) since $A_{p}$ is not $\alpha$-thin at infinity. By the linearity of balayage and (7.16) we actually have $\tilde{\boldsymbol{v}} \in \mathscr{E}_{\alpha}^{\sigma}\left(\mathbf{A}, \mathbf{a} ; \mathbb{R}^{n}\right)$, and finally $\tilde{\boldsymbol{v}} \in \mathscr{E}_{\alpha, \mathbf{f}} \boldsymbol{\sigma}\left(\mathbf{A}, \mathbf{a} ; \mathbb{R}^{n}\right)$ by (5.2). In consequence of (4.4), (5.2) and (7.20) we therefore obtain

$$
\begin{aligned}
G_{\alpha, \mathbf{f}}(\boldsymbol{v}) & =\kappa_{\alpha}(\boldsymbol{v}, \boldsymbol{v})+2\left\langle\mathbf{f}^{+}, \boldsymbol{v}^{+}\right\rangle=\left\|R_{\mathbf{A}} \boldsymbol{v}^{+}-v^{p}\right\|_{\alpha}^{2}+2\left\langle\mathbf{f}^{+}, \boldsymbol{v}^{+}\right\rangle \\
& \geqslant\left\|R_{\mathbf{A}} \boldsymbol{v}^{+}-\left(R_{\mathbf{A}} \boldsymbol{v}^{+}\right)^{\prime}\right\|_{\alpha}^{2}+2\left\langle\mathbf{f}^{+}, \boldsymbol{v}^{+}\right\rangle=G_{\alpha, \mathbf{f}}(\tilde{\boldsymbol{v}}) \geqslant G_{\alpha, \mathbf{f}}^{\boldsymbol{\sigma}}\left(\mathbf{A}, \mathbf{a} ; \mathbb{R}^{n}\right),
\end{aligned}
$$

which establishes (7.17) in view of the arbitrary choice of $\boldsymbol{v} \in \mathscr{E}_{\alpha, \mathbf{f}}^{\xi}\left(\mathbf{A}, \mathbf{a} ; \mathbb{R}^{n}\right)$.

Assuming now that (5.9) holds, we next prove (7.18). It is clear from (7.17) and (7.19) that $\mathfrak{\Xi}_{\alpha, \mathbf{f}}^{\sigma}\left(\mathbf{A}, \mathbf{a} ; \mathbb{R}^{n}\right) \subset \mathfrak{\Xi}_{\alpha, \mathbf{f}}^{\xi}\left(\mathbf{A}, \mathbf{a} ; \mathbb{R}^{n}\right)$. As for the converse inclusion, fix $\lambda=\left(\lambda^{i}\right)_{i \in I} \in \mathfrak{S}_{\alpha, \mathbf{f}}^{\xi}\left(\mathbf{A}, \mathbf{a} ; \mathbb{R}^{n}\right)$. Then $\lambda^{p}=\left(R_{\mathbf{A}} \lambda^{+}\right)^{\prime}$ by (6.3), and in the same manner as in the preceding paragraph we get $\lambda \in \mathscr{E}_{\alpha, \mathbf{f}}^{\boldsymbol{\sigma}}\left(\mathbf{A}, \mathbf{a} ; \mathbb{R}^{n}\right)$. Hence, $\lambda \in \mathfrak{S}_{\alpha, \mathbf{f}}^{\sigma}\left(\mathbf{A}, \mathbf{a} ; \mathbb{R}^{n}\right)$, for $G_{\alpha, \mathbf{f}}(\lambda)=G_{\alpha, \mathbf{f}}^{\xi}\left(\mathbf{A}, \mathbf{a} ; \mathbb{R}^{n}\right)=G_{\alpha, \mathbf{f}}^{\sigma}\left(\mathbf{A}, \mathbf{a} ; \mathbb{R}^{n}\right)$ by (7.17).

Thus, the theory of weighted minimum $\alpha$-Riesz energy problems with a constraint $\xi$ given by (5.8) and acting only on measures carried by the $A_{j}, j \in I^{+}$, developed in Section 7.1, remains valid in its full generality for the constraint $\boldsymbol{\sigma}$, defined by (7.16) and acting on all the components of $\boldsymbol{\mu} \in \mathscr{E}_{\alpha}^{+}\left(\mathbf{A}, \mathbf{a} ; \mathbb{R}^{n}\right)$.

\section{Proofs of Theorems 7.1 and $\mathbf{7 . 2}$}

Observe that, if Case II takes place, then

$$
\begin{gathered}
\zeta \in \mathscr{E}_{g}(D), \\
f_{j}=\kappa_{\alpha}\left(\cdot, \zeta-\zeta^{\prime}\right)=g(\cdot, \zeta) \quad c_{g} \text {-n.e. on } D \text { for all } j \in I^{+} .
\end{gathered}
$$

Indeed, (8.1) is obvious by (4.7), and (8.2) holds by Lemma 4.1 and Remark 4.1. By (8.1) and (8.2), in Case II for every $\boldsymbol{v} \in \mathscr{E}_{g}^{+}\left(\mathbf{A}^{+} ; D\right)$ we get 


$$
\begin{aligned}
G_{g, \mathbf{f}^{+}}(\boldsymbol{v}) & =\left\|R_{\mathbf{A}^{+}} \boldsymbol{v}\right\|_{g}^{2}+2 \sum_{j \in I^{+}} g\left(\zeta, v^{j}\right) \\
& =\left\|R_{\mathbf{A}^{+}} \boldsymbol{v}\right\|_{g}^{2}+2 g\left(\zeta, R_{\mathbf{A}^{+}} \boldsymbol{v}\right)=\left\|R_{\mathbf{A}^{+}} \boldsymbol{v}+\zeta\right\|_{g}^{2}-\|\zeta\|_{g}^{2} .
\end{aligned}
$$

8.1. Proof of Theorem 7.1. By Theorem 6.1, Theorem 7.1 will be proved once we have established the following assertion.

THEOREM 8.1. Under the assumptions of Theorem 7.1, Problem 3.2 for D, $g, \mathbf{A}^{+}, \mathbf{a}^{+}, \mathbf{f}^{+}$and $\xi^{+}$is solvable, i.e. there is $\boldsymbol{\mu} \in \mathscr{E}_{g, \mathbf{f}^{+}}^{\xi^{+}}\left(\mathbf{A}^{+}, \mathbf{a}^{+} ; D\right)$ with

$$
G_{g, \mathbf{f}^{+}}(\boldsymbol{\mu})=G_{g, \mathbf{f}^{+}}^{\xi^{+}}\left(\mathbf{A}^{+}, \mathbf{a}^{+} ; D\right) .
$$

Proof. Note that Problem 3.2 for $D, g, \mathbf{A}^{+}, \mathbf{a}^{+}, \mathbf{f}^{+}$and $\boldsymbol{\xi}^{+}$makes sense since by assumption (5.9) and identity (6.2) we have

$$
G_{g, \mathbf{f}^{+}}^{\xi^{+}}\left(\mathbf{A}^{+}, \mathbf{a}^{+} ; D\right)<\infty .
$$

Actually, $G_{g, \mathbf{f}^{+}}^{\xi^{+}}\left(\mathbf{A}^{+}, \mathbf{a}^{+} ; D\right)$ is finite, which is clear from (6.2) (cf. Remark 5.1). In view of $(8.4)$, there is a sequence $\left\{\boldsymbol{\mu}_{k}\right\}_{k \in \mathbb{N}} \subset \mathscr{E}_{g, \mathbf{f}^{+}}^{\xi^{+}}\left(\mathbf{A}^{+}, \mathbf{a}^{+} ; D\right)$ such that

$$
\lim _{k \rightarrow \infty} G_{g, \mathbf{f}^{+}}\left(\boldsymbol{\mu}_{k}\right)=G_{g, \mathbf{f}^{+}}^{\xi^{+}}\left(\mathbf{A}^{+}, \mathbf{a}^{+} ; D\right) .
$$

Since the $\alpha$-Green kernel $g$ satisfies the energy principle [15, Theorem 4.9], $\mathscr{E}_{g}(D)$ forms a pre-Hilbert space with the inner product $g\left(v, v_{1}\right)$ and the energy norm $\|v\|_{g}=\sqrt{g(v, v)}$. Furthermore, $\mathscr{E}_{g, \mathbf{f}^{+}}^{\xi^{+}}\left(\mathbf{A}^{+}, \mathbf{a}^{+} ; D\right)$ is convex and $R_{\mathbf{A}^{+}}$is an isometric mapping between the semimetric space $\mathscr{E}_{g}^{+}\left(\mathbf{A}^{+} ; D\right)$ and its $R_{\mathbf{A}^{+}}$-image into $\mathscr{E}_{g}(D)$ (see Theorem 3.1). We are therefore able to apply to the set $\left\{\boldsymbol{\mu}_{k}: k \in \mathbb{N}\right\}$ arguments similar to those in the proof of Lemma 3.6, and we get

$$
0 \leqslant\left\|R_{\mathbf{A}^{+}} \boldsymbol{\mu}_{k}-R_{\mathbf{A}^{+}} \boldsymbol{\mu}_{\ell}\right\|_{g}^{2} \leqslant-4 G_{g, \mathbf{f}^{+}}^{\xi^{+}}\left(\mathbf{A}^{+}, \mathbf{a}^{+} ; D\right)+2 G_{g, \mathbf{f}^{+}}\left(\boldsymbol{\mu}_{k}\right)+2 G_{g, \mathbf{f}^{+}}\left(\boldsymbol{\mu}_{\ell}\right) .
$$

Letting here $k, \ell \rightarrow \infty$ and combining the relation thus obtained with (8.5), we see in view of the finiteness of $G_{g, \mathbf{f}^{+}}^{\xi^{+}}\left(\mathbf{A}^{+}, \mathbf{a}^{+} ; D\right)$ that $\left\{R_{\mathbf{A}^{+}} \boldsymbol{\mu}_{k}\right\}_{k \in \mathbb{N}}$ forms a strong Cauchy sequence in the metric space $\mathscr{E}_{g}^{+}(D)$. In particular, this implies

$$
\sup _{k \in \mathbb{N}}\left\|R_{\mathbf{A}^{+}} \boldsymbol{\mu}_{k}\right\|_{g}<\infty
$$

Since the sets $A_{j}, j \in I^{+}$, are (relatively) closed in $D$, the cones $\mathfrak{M}^{\xi^{j}}\left(A_{j} ; D\right)$, $j \in I^{+}$, are vaguely closed in $\mathfrak{M}(D)$, and therefore $\mathfrak{M}^{\xi^{+}}\left(\mathbf{A}^{+} ; D\right)$ is vaguely closed in $\mathfrak{M}(D)^{p-1}$ (cf. Definition 3.3). Furthermore, $\mathfrak{M}^{\xi^{+}}\left(\mathbf{A}^{+}, \mathbf{a}^{+} ; D\right)$ is vaguely bounded, hence vaguely relatively compact by Lemma 3.4. Thus, there is a vague cluster point $\boldsymbol{\mu}$ of the sequence $\left\{\boldsymbol{\mu}_{k}\right\}_{k \in \mathbb{N}}$ chosen above, which 
belongs to $\mathfrak{M}^{\xi^{+}}\left(\mathbf{A}^{+} ; D\right)$. Passing to a subsequence and changing notations, we assume that

$$
\boldsymbol{\mu}_{k} \rightarrow \boldsymbol{\mu} \quad \text { vaguely as } k \rightarrow \infty .
$$

We assert that the $\boldsymbol{\mu}$ is a solution to Problem 3.2 for $D, g, \mathbf{A}^{+}, \mathbf{a}^{+}, \mathbf{f}^{+}$and $\boldsymbol{\xi}^{+}$.

Fix $j \in I^{+}$. Applying Lemma 2.1 to $1_{D} \in \Psi(D)$, we obtain from (8.7)

$$
\mu^{j}(D) \leqslant \lim _{k \rightarrow \infty} \mu_{k}^{j}(D)=a_{j}
$$

We proceed by showing that equality prevails in the inequality here, and hence

$$
\boldsymbol{\mu} \in \mathfrak{M}^{\xi^{+}}\left(\mathbf{A}^{+}, \mathbf{a}^{+} ; D\right) .
$$

Consider an exhaustion of $A_{j}$ by an increasing sequence $\left\{K_{\ell}\right\}_{\ell \in \mathbb{N}}$ of compact subsets of $D$. Since $1_{K_{\ell}}$ is upper semicontinuous on $D$ (and of course bounded), we get from Lemma 2.1 (with $\psi=-1_{K_{\ell}}$ )

$$
\begin{aligned}
a_{j} & \geqslant \mu^{j}(D)=\lim _{\ell \rightarrow \infty} \mu^{j}\left(K_{\ell}\right) \geqslant \lim _{\ell \rightarrow \infty} \limsup _{k \rightarrow \infty} \mu_{k}^{j}\left(K_{\ell}\right) \\
& =a_{j}-\lim _{\ell \rightarrow \infty} \liminf _{k \rightarrow \infty} \mu_{k}^{j}\left(A_{j} \backslash K_{\ell}\right) .
\end{aligned}
$$

Thus, (8.8) will follow if we show that

$$
\lim _{\ell \rightarrow \infty} \liminf _{k \rightarrow \infty} \mu_{k}^{j}\left(A_{j} \backslash K_{\ell}\right)=0 .
$$

By (7.1),

$$
\infty>\xi^{j}(D)=\lim _{\ell \rightarrow \infty} \xi^{j}\left(K_{\ell}\right)
$$

and therefore

$$
\lim _{\ell \rightarrow \infty} \xi^{j}\left(A_{j} \backslash K_{\ell}\right)=0 .
$$

Combined with $\mu_{k}^{j}\left(A_{j} \backslash K_{\ell}\right) \leqslant \xi^{j}\left(A_{j} \backslash K_{\ell}\right)$ for all $k, \ell \in \mathbb{N}$, this implies (8.9), and consequently (8.8).

Furthermore, since $R_{\mathbf{A}^{+}} \boldsymbol{\mu}_{k} \rightarrow R_{\mathbf{A}^{+}} \boldsymbol{\mu}$ vaguely in $\mathfrak{M}^{+}(D)$, it is seen from [3, Chapter III, Section 5, Exercise 5] that $R_{\mathbf{A}^{+}} \boldsymbol{\mu}_{k} \otimes R_{\mathbf{A}^{+}} \boldsymbol{\mu}_{k} \rightarrow R_{\mathbf{A}^{+}} \boldsymbol{\mu} \otimes R_{\mathbf{A}^{+}} \boldsymbol{\mu}$ vaguely in $\mathfrak{M}^{+}(D \times D)$. Applying Lemma 2.1 to $X=D \times D$ and $\psi=g$, we get

$$
g\left(R_{\mathbf{A}^{+}} \boldsymbol{\mu}, R_{\mathbf{A}^{+}} \boldsymbol{\mu}\right) \leqslant \liminf _{k \rightarrow \infty}\left\|R_{\mathbf{A}^{+}} \boldsymbol{\mu}_{k}\right\|_{g}^{2}<\infty
$$

the latter inequality being valid by (8.6). Hence, $\boldsymbol{\mu} \in \mathscr{E}_{g}^{+}\left(\mathbf{A}^{+} ; D\right)$ (cf. (3.8)). Combined with $(8.8)$, this yields $\boldsymbol{\mu} \in \mathscr{E}_{g}^{\xi^{+}}\left(\mathbf{A}^{+}, \mathbf{a}^{+} ; D\right)$. Note that $G_{g, \mathbf{f}^{+}}(\boldsymbol{\mu})>$ 
$-\infty$, which is clear from (8.3) if Case II takes place, and otherwise (in the presence of Case I) it is obvious by $f_{j} \geqslant 0, j \in I^{+}$. The claimed assertion $\boldsymbol{\mu} \in \mathfrak{S}_{q, \mathbf{f}^{+}}^{\xi^{+}}\left(\mathbf{A}^{+}, \mathbf{a}^{+} ; D\right)$ will therefore be established once we have shown that

$$
G_{g, \mathbf{f}^{+}}(\boldsymbol{\mu}) \leqslant \lim _{k \rightarrow \infty} G_{g, \mathbf{f}^{+}}\left(\boldsymbol{\mu}_{k}\right) .
$$

Since the kernel $g$ is perfect [15, Theorem 4.11], the sequence $\left\{R_{\mathbf{A}^{+}} \boldsymbol{\mu}_{k}\right\}_{k \in \mathbb{N}}$, being strong Cauchy in $\mathscr{E}_{g}^{+}(D)$ and vaguely convergent to $R_{\mathbf{A}^{+}} \boldsymbol{\mu}$, converges to the same limit strongly in $\mathscr{E}_{g}^{+}(D)$, i.e.

$$
\lim _{k \rightarrow \infty}\left\|R_{\mathbf{A}^{+}} \boldsymbol{\mu}_{k}-R_{\mathbf{A}^{+}} \boldsymbol{\mu}\right\|_{g}=0
$$

which in view of (3.8) and (3.10) is equivalent to the relation

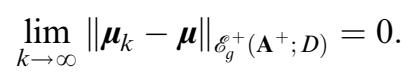

Also note that the mapping $\boldsymbol{v} \mapsto G_{g, \mathbf{f}^{+}}(\boldsymbol{v})$ is vaguely 1.s.c., resp. strongly continuous, on $\mathscr{E}_{q, \mathbf{f}^{+}}^{+}\left(\mathbf{A}^{+} ; D\right)$ if Case I, resp. Case II, takes place. In fact, since $g(\boldsymbol{v}, \boldsymbol{v})$ is vaguely l.s.c. on $\mathscr{E}_{g}^{+}\left(\mathbf{A}^{+} ; D\right)$, the former assertion follows from Lemma 2.1. As for the latter assertion, it is obvious by (8.3). In view of this observation, (8.7) and (8.11) result in (8.10).

COROLlary 8.1. Suppose that the assumptions of Theorem 7.1 are fulfilled. Then the (nonempty) class $\mathfrak{S}_{g, \mathbf{f}^{+}}^{\xi^{+}}\left(\mathbf{A}^{+}, \mathbf{a}^{+} ; D\right)$ of all solutions to Problem 3.2 for $D, g, \mathbf{A}^{+}, \mathbf{a}^{+}, \mathbf{f}^{+}$and $\boldsymbol{\xi}^{+}$is vaguely compact in $\mathfrak{M}(D)^{p-1}$.

Proof. Any $\boldsymbol{\mu}_{k} \in \mathfrak{S}_{g, \mathbf{f}^{+}}^{\xi^{+}}\left(\mathbf{A}^{+}, \mathbf{a}^{+} ; D\right), k \in \mathbb{N}$, form a strong Cauchy sequence in $\mathscr{E}_{g}^{+}\left(\mathbf{A}^{+} ; D\right)$ according to Lemma 3.6. Furthermore, the set $\left\{\boldsymbol{\mu}_{k}: k \in \mathbb{N}\right\}$ is vaguely closed and relatively compact in $\mathfrak{M}(D)^{p-1}$ (see Section 3.3 with $X=D)$. Therefore in the same manner as in the proof of Theorem 8.1 we see that any vague cluster point of $\left\{\boldsymbol{\mu}_{k}\right\}_{k \in \mathbb{N}}$ belongs to $\mathfrak{S}_{g, \mathbf{f}^{+}}^{\xi^{+}}\left(\mathbf{A}^{+}, \mathbf{a}^{+} ; D\right)$.

8.2. Proof of Theorem 7.2. Assume that the requirements of the theorem are fulfilled. Since Case II with $\zeta \geqslant 0$ takes place, we get from (8.1) and $(8.2)$

$$
G_{g,\left.f_{1}\right|_{D}}(v)=\|v\|_{g}^{2}+2 g(\zeta, v) \in[0, \infty) \quad \text { for all } v \in \mathscr{E}_{g}^{+}\left(A_{1} ; D\right)
$$

Consider numbers $r_{\ell}>0, \ell \in \mathbb{N}$, such that $r_{\ell} \uparrow \infty$ as $\ell \rightarrow \infty$, and write $B_{r_{\ell}}:=B\left(0, r_{\ell}\right), A_{1, r_{\ell}}:=A_{1} \cap B_{r_{\ell}}$. Since $c_{\alpha}\left(A_{1}\right)=\infty$ by assumption and since $c_{\alpha}\left(B_{r_{\ell}}\right)<\infty$ for every $\ell \in \mathbb{N}$, we infer from the subadditivity of $c_{\alpha}(\cdot)$ on universally measurable sets [11, Lemma 2.3.5] that $c_{\alpha}\left(A_{1} \backslash B_{r_{\ell}}\right)=\infty$. Hence 
for every $\ell \in \mathbb{N}$ there is $\xi_{\ell} \in \mathscr{E}_{\alpha}^{+}\left(A_{1} \backslash B_{r_{\ell}}, a_{1} ; \mathbb{R}^{n}\right)$ of compact support $S_{D}^{\xi_{\ell}}$ such that

$$
\left\|\xi_{\ell}\right\|_{\alpha} \leqslant \ell^{-2}
$$

Clearly, the $r_{\ell}$ can be chosen successively so that $A_{1, r_{\ell}} \cup S_{D}^{\xi_{\ell}} \subset A_{1, r_{\ell+1}}$. Any compact set $K \subset \mathbb{R}^{n}$ is contained in a ball $B_{r_{\ell_{0}}}$ with $\ell_{0}$ large enough, and hence $K$ has points in common with only finitely many $S_{D}^{\xi_{l}}$. Therefore, $\xi^{1}$ defined by

$$
\xi^{1}(\varphi):=\sum_{\ell \in \mathbb{N}} \xi_{\ell}(\varphi) \quad \text { for any } \varphi \in C_{0}\left(\mathbb{R}^{n}\right)
$$

is a positive Radon measure on $\mathbb{R}^{n}$ carried by $A_{1}$. Furthermore, $\xi^{1}\left(A_{1}\right)=\infty$ and $\xi^{1} \in \mathscr{E}_{\alpha}^{+}\left(\mathbb{R}^{n}\right)$. To prove the latter, note that $\eta_{k}:=\xi_{1}+\cdots+\xi_{k} \in \mathscr{E}_{\alpha}^{+}\left(\mathbb{R}^{n}\right)$, which is clear from (8.13) and the triangle inequality in $\mathscr{E}_{\alpha}\left(\mathbb{R}^{n}\right)$. Also observe that $\eta_{k} \rightarrow \xi^{1}$ vaguely because for any $\varphi \in C_{0}\left(\mathbb{R}^{n}\right)$ there is $k_{0}$ such that $\xi^{1}(\varphi)=\eta_{k}(\varphi)$ for all $k \geqslant k_{0}$. As $\left\|\eta_{k}\right\|_{\alpha} \leqslant L:=\sum_{\ell \in \mathbb{N}^{\ell}} \ell^{-2}<\infty$ for all $k \in \mathbb{N}$, Lemma 2.1 with $X=\mathbb{R}^{n} \times \mathbb{R}^{n}$ and $\psi=\kappa_{\alpha}$ yields $\left\|\xi^{1}\right\|_{\alpha} \leqslant L$.

Each $\xi_{\ell}$ belongs to $\mathscr{E}_{g}^{+}\left(A_{1}, a_{1} ; D\right)$ and moreover, by (4.10) and (8.13),

$$
\left\|\xi_{\ell}\right\|_{g} \leqslant\left\|\xi_{\ell}\right\|_{\alpha} \leqslant \ell^{-2} \text {. }
$$

As Case II with $\zeta \geqslant 0$ takes place, $\xi_{\ell} \in \mathscr{E}_{g,\left.f_{1}\right|_{D}}^{\xi^{1}}\left(A_{1}, a_{1} ; D\right)$ for all $\ell \in \mathbb{N}$ by (8.12). Therefore, by the Cauchy-Schwarz (Bunyakovski) inequality in $\mathscr{E}_{g}(D)$,

$$
0 \leqslant G_{g,\left.f_{1}\right|_{D}}^{\xi^{1}}\left(A_{1}, a_{1} ; D\right) \leqslant \lim _{\ell \rightarrow \infty}\left[\left\|\xi_{\ell}\right\|_{g}^{2}+2 g\left(\zeta, \xi_{\ell}\right)\right] \leqslant 2\|\zeta\|_{g} \lim _{\ell \rightarrow \infty}\left\|\xi_{\ell}\right\|_{g}=0
$$

where the first and the second inequalities hold by (8.12), and the third inequality and the equality are valid by (8.14). Hence, $G_{g,\left.f_{1}\right|_{D}}^{\xi^{1}}\left(A_{1}, a_{1} ; D\right)=0$. As seen from (8.12), such infimum can be attained only at zero measure, which is impossible because $0 \notin \mathscr{E}_{g,\left.f_{1}\right|_{D}}^{\xi^{1}}\left(A_{1}, a_{1} ; D\right)$. Application of Theorem 6.1 completes the proof.

\section{Proofs of Theorems 7.3, 7.4 and 7.5}

Throughout this section we maintain all the requirements on $\mathbf{A}, \mathbf{a}, \mathbf{f}$, and $\boldsymbol{\xi}$ imposed at the beginning of Section 5.2, except for (5.9) which follows automatically from the hypotheses of the assertions under proving in view of Lemma 5.2.

9.1. Proof of Theorem 7.3. Fix $\lambda_{\mathbf{A}} \in \mathscr{E}_{\alpha, \mathbf{f}}^{+}\left(\mathbf{A}, \mathbf{a} ; \mathbb{R}^{n}\right)$. Then each $\lambda_{\mathbf{A}}^{i}, i \in I$, has finite $\alpha$-Riesz energy, and hence it is $c_{\alpha}$-absolutely continuous. Note that, since 
$f_{p}=0$ n.e. on $A_{p},(7.4)$ can alternatively be rewritten as $\kappa_{\alpha}^{\lambda_{\mathrm{A}}, p}=0$ n.e. on $A_{p}$, which by (3.9) (with $X=\mathbb{R}^{n}$ and $\kappa=\kappa_{\alpha}$ ) is equivalent to the relation

$$
\kappa_{\alpha}\left(\cdot, R_{\mathbf{A}} \lambda_{\mathbf{A}}^{+}-\lambda^{p}\right)=0 \quad \text { n.e. on } A_{p} .
$$

In view of the characteristic property (4.2) of the swept measures, this shows that for the given $\lambda_{\mathbf{A}},(7.4)$ and (6.3) are equivalent. On account of Theorem 6.1, we thus see that when proving the equivalence of assertions (i) and (ii) of Theorem 7.3, there is no loss of generality in assuming $\lambda_{\mathrm{A}}$ to satisfy (6.3).

Substituting (6.3) into (3.9), we therefore get for every $i \in I$

$$
\kappa_{\alpha}^{\lambda_{\mathbf{A}}, i}(\cdot)=s_{i} \kappa_{\alpha}\left(\cdot, R_{\mathbf{A}} \lambda_{\mathbf{A}}^{+}-\left(R_{\mathbf{A}} \lambda_{\mathbf{A}}^{+}\right)^{\prime}\right) \quad \text { n.e. on } \mathbb{R}^{n} .
$$

In particular, for every $j \in I^{+}$we have

$$
\kappa_{\alpha}^{\lambda_{\mathbf{A}}, j}(\cdot)=g\left(\cdot, R_{\mathbf{A}^{+}} \lambda_{\mathbf{A}}^{+}\right)=g^{\lambda_{\mathbf{A}}^{+}, j}(\cdot) \quad \text { n.e. on } D
$$

and hence, by (3.12),

$$
W_{\alpha, \mathbf{f}}^{\lambda_{\mathbf{A}}, j}=W_{g, \mathbf{f}^{+}}^{\lambda_{\mathbf{A}}^{+}, j} \quad \text { n.e. on } D \text {. }
$$

(Note that (9.2) has been obtained from (9.1) with the aid of (4.6), applied to $R_{\mathbf{A}^{+}} \lambda_{\mathbf{A}}^{+}$in place of $\mu$, and (3.9), the latter with $X=D$ and $\kappa=g$.)

If Case II holds, then for every $i \in I$ we also get from (9.1) and

$$
W_{\alpha, \mathbf{f}}^{\lambda_{\mathbf{A}}, i}(\cdot)=s_{i} \kappa_{\alpha}\left(\cdot,\left(R_{\mathbf{A}} \lambda_{\mathbf{A}}^{+}+\zeta\right)-\left(R_{\mathbf{A}} \lambda_{\mathbf{A}}^{+}+\zeta\right)^{\prime}\right) \quad \text { n.e. on } \mathbb{R}^{n} .
$$

By [15, Corollary 3.14], the function on the right (hence, also that on the left) in this relation takes the value 0 at every $\alpha$-regular point of $A_{p}$, which gives (7.5).

By Theorem 6.1, what has been shown just above yields that Theorem 7.3 will be proved once the following theorem has been established.

THEOREM 9.1. Under the hypotheses of Theorem 7.3 the following two assertions are equivalent for any $\lambda \in \underset{g}{\mathscr{E} \xi^{+}} \mathbf{f}^{+}\left(\mathbf{A}^{+}, \mathbf{a}^{+} ; D\right)$ :

(i') $\lambda \in \mathbb{S}_{g, \mathbf{f}^{+}}^{\xi^{+}}\left(\mathbf{A}^{+}, \mathbf{a}^{+} ; D\right)$.

(ii') There is a vector $\left(c_{j}\right)_{j \in I^{+}} \in \mathbb{R}^{p-1}$ such that for every $j \in I^{+}$

$$
\begin{aligned}
& W_{g, \mathbf{f}^{+}}^{\lambda,} \geqslant c_{j} \quad\left(\xi^{j}-\lambda^{j}\right) \text {-a.e. }, \\
& W_{g, \mathbf{f}^{+}}^{\lambda, j} \leqslant c_{j} \quad \lambda^{j} \text {-a.e. }
\end{aligned}
$$

Proof. Suppose first that (i') holds. To verify (ii'), fix $j \in I^{+}$. For every $\boldsymbol{\mu}=\left(\mu^{\ell}\right)_{\ell \in I^{+}} \in \mathscr{E}_{g, \mathbf{f}^{+}}^{\xi^{+}}\left(\mathbf{A}^{+}, \mathbf{a}^{+} ; D\right)$ write $\boldsymbol{\mu}_{j}:=\left(\mu_{j}^{\ell}\right)_{\ell \in I^{+}}$, where $\mu_{j}^{\ell}:=\mu^{\ell}$ for all $\ell \neq j$ and $\mu_{j}^{j}=0$; then $\boldsymbol{\mu}_{j} \in \mathscr{E}_{g, \mathbf{f}^{+}}^{+}\left(\mathbf{A}^{+} ; D\right)$. Also define $\tilde{f}_{j}:=\left.f_{j}\right|_{D}+g^{\lambda_{j}, j}$; by 
substituting (3.6) with $\kappa=g$ we then obtain

$$
\tilde{f}_{j}=\left.f_{j}\right|_{D}+\sum_{\ell \in I^{+}, \ell \neq j} g\left(\cdot, \lambda^{\ell}\right) .
$$

Since $g\left(\cdot, \lambda^{\ell}\right)>0$ on $D$ for all $\ell \in I^{+}$according to [15, Lemma 4.1] and since $f_{j}$ is lower bounded on $A_{j}$ by assumption, the function

$$
W_{g, \tilde{f}_{j}}^{\lambda^{j}}:=g\left(\cdot, \lambda^{j}\right)+\tilde{f}_{j}, \quad j \in I^{+},
$$

is likewise lower bounded on $A_{j}$. Furthermore, both $\tilde{f}_{j}$ and $W_{g, \tilde{f}_{j}}^{\lambda^{j}}$ are finite n.e. on the set $A_{j}^{\circ}$, which is clear from (5.10) and Lemma 3.2.

Applying (3.7) and (3.13), we get for any $\boldsymbol{\mu} \in \mathscr{E}_{g, \mathbf{f}^{+}}^{\xi^{+}}\left(\mathbf{A}^{+}, \mathbf{a}^{+} ; D\right)$ with the additional property that $\boldsymbol{\mu}_{j}=\lambda_{j}$ (in particular for $\boldsymbol{\mu}=\boldsymbol{g}=\boldsymbol{\lambda}$ )

$$
G_{g, \mathbf{f}^{+}}(\boldsymbol{\mu})=G_{g, \mathbf{f}^{+}}\left(\lambda_{j}\right)+G_{g, \tilde{f}_{j}}\left(\mu^{j}\right) .
$$

Combined with $G_{g, \mathbf{f}^{+}}(\boldsymbol{\mu}) \geqslant G_{g, \mathbf{f}^{+}}(\lambda)$, this yields $G_{g, \tilde{f}_{j}}\left(\mu^{j}\right) \geqslant G_{g, \tilde{f}_{j}}\left(\lambda^{j}\right)$. Hence, $\lambda^{j}$ is the (unique) solution to the problem of minimizing $G_{g, \tilde{f}_{j}}(v)$, where $v$ ranges over $\mathscr{E}_{g, \tilde{f}_{j}}^{\xi^{j}}\left(A_{j}, a_{j} ; D\right)$. This enables us to show that there is $c_{j} \in \mathbb{R}$ such that

$$
\begin{aligned}
& W_{g, \tilde{f}_{j}}^{\lambda^{j}} \geqslant c_{j} \quad\left(\xi^{j}-\lambda^{j}\right) \text {-a.e. } \\
& W_{g, \tilde{f}_{j}}^{\lambda^{j}} \leqslant c_{j} \quad \lambda^{j} \text {-a.e. }
\end{aligned}
$$

In doing this we shall use permanently the fact that both $\xi^{j}$ and $\lambda^{j}$ have finite $\alpha$-Riesz energy, and hence they are $c_{\alpha}$-absolutely continuous.

Indeed, (9.7) holds with

$$
c_{j}:=L_{j}:=\sup \left\{t \in \mathbb{R}: W_{g, \tilde{f}_{j}}^{\lambda^{j}} \geqslant t\left(\xi^{j}-\lambda^{j}\right) \text {-a.e. }\right\} .
$$

In turn, (9.7) with $c_{j}=L_{j}$ implies that $L_{j}<\infty$ because $W_{g, \tilde{f}_{j}}^{\lambda^{j}}<\infty$ holds n.e. on $A_{j}^{\circ}$ and hence $\left(\xi^{j}-\lambda^{j}\right)$-a.e. on $A_{j}^{\circ}$, while $\left(\xi^{j}-\lambda^{j}\right)\left(A_{j}^{\circ}\right) \stackrel{g, f_{j}}{>} 0$ by (5.11). Also $L_{j}>-\infty$, for $W_{g, \tilde{f}_{j}}^{\lambda^{j}}$ is lower bounded on $A_{j}$ (see above).

We next establish (9.8) with $c_{j}=L_{j}$. To this end, write for any $w \in \mathbb{R}$

$$
A_{j}^{+}(w):=\left\{x \in A_{j}: W_{g, \tilde{f}_{j}}^{\lambda^{j}}(x)>w\right\}, \quad A_{j}^{-}(w):=\left\{x \in A_{j}: W_{g, \tilde{f}_{j}}^{\lambda^{j}}(x)<w\right\} .
$$

On the contrary, let (9.8) with $c_{j}=L_{j}$ fail, i.e. $\lambda^{j}\left(A_{j}^{+}\left(L_{j}\right)\right)>0$. Since $W_{g, \tilde{f}_{j}}^{\lambda^{j}}$ is $\lambda^{j}$-measurable and $L_{j}$ is finite, one can choose $w_{j} \in\left(L_{j}, \infty\right)$ so that $\lambda^{j}\left(A_{j}^{+}\left(w_{j}\right)\right)>0$. At the same time, as $w_{j}>L_{j}$, it follows from the definition of $L_{j}$ that $\left(\xi^{j}-\lambda^{j}\right)\left(A_{j}^{-}\left(w_{j}\right)\right)>0$. Therefore, there exist compact sets $K_{1} \subset A_{j}^{+}\left(w_{j}\right)$ and $K_{2} \subset A_{j}^{-}\left(w_{j}\right)$ such that

$$
0<\lambda^{j}\left(K_{1}\right)<\left(\xi^{j}-\lambda^{j}\right)\left(K_{2}\right) .
$$


Write $\tau^{j}:=\left.\left(\xi^{j}-\lambda^{j}\right)\right|_{K_{2}}$; then $g\left(\tau^{j}, \tau^{j}\right)<\kappa_{\alpha}\left(\tau^{j}, \tau^{j}\right)<\infty$, where the former inequality holds by (4.10). As $\left\langle W_{g, \tilde{f}_{j}}^{\lambda^{j}}, \tau^{j}\right\rangle \leqslant w_{j} \tau^{j}\left(K_{2}\right)<\infty$, we therefore get $\left\langle\tilde{f}_{j}, \tau^{j}\right\rangle<\infty$. Define $\theta^{j}:=\lambda^{j}-\left.\lambda^{j}\right|_{K_{1}}+b_{j} \tau^{j}$, where $b_{j}:=\lambda^{j}\left(K_{1}\right) / \tau^{j}\left(K_{2}\right) \in$ $(0,1)$ by $(9.9)$. Straightforward verification then shows that $\theta^{j}\left(A_{j}\right)=a_{j}$ and $\theta^{j} \leqslant \xi^{j}$, and hence $\theta^{j} \in \mathscr{E}_{g, \tilde{f}_{j}}^{\mathscr{f}^{j}}\left(A_{j}, a_{j} ; D\right)$. On the other hand,

$$
\begin{aligned}
\left\langle W_{g, \tilde{f}_{j}}^{\lambda^{j}}, \theta^{j}-\lambda^{j}\right\rangle & =\left\langle W_{g, \tilde{f}_{j}}^{\lambda^{j}}-w_{j}, \theta^{j}-\lambda^{j}\right\rangle \\
& =-\left\langle W_{g, \tilde{f}_{j}}^{\lambda^{j}}-w_{j},\left.\lambda^{j}\right|_{K_{1}}\right\rangle+b_{j}\left\langle W_{g, \tilde{f}_{j}}^{\lambda^{j}}-w_{j}, \tau^{j}\right\rangle<0,
\end{aligned}
$$

which is impossible by (the scalar version of) Lemma 3.5 with the (convex) set $\mathfrak{E}=\mathscr{E}_{g, \tilde{f}_{j}}^{\tilde{\xi}^{j}}\left(A_{j}, a_{j} ; D\right)$. This contradiction establishes (9.8).

Substituting (9.5) into (9.6) and then comparing the result obtained with (3.6) and (3.12), we see that

$$
W_{g, \tilde{f}_{j}}^{\lambda^{j}}=W_{g, \mathbf{f}^{+}}^{\lambda, j}
$$

Combined with (9.7) and (9.8), this establishes (9.3) and (9.4), thus completing the proof that $\left(\mathrm{i}^{\prime}\right)$ implies $\left(\mathrm{ii}^{\prime}\right)$.

Conversely, let (ii') hold. On account of (9.10), for every $j \in I^{+}$relations (9.7) and (9.8) are then fulfilled with $\tilde{f}_{j}$ defined by (9.5). This yields

$$
\lambda^{j}\left(A_{j}^{+}\left(c_{j}\right)\right)=0 \quad \text { and } \quad\left(\xi^{j}-\lambda^{j}\right)\left(A_{j}^{-}\left(c_{j}\right)\right)=0 .
$$

For any $\boldsymbol{v} \in \mathscr{E}_{g, \mathbf{f}^{+}}^{\xi^{+}}\left(\mathbf{A}^{+}, \mathbf{a}^{+} ; D\right)$ we therefore get

$$
\begin{aligned}
\left\langle W_{g, \mathbf{f}^{+}}^{\lambda, j}, v^{j}-\lambda^{j}\right\rangle & =\left\langle W_{g, \tilde{f}_{j}}^{\lambda^{j}}-c_{j}, v^{j}-\lambda^{j}\right\rangle \\
& =\left\langle W_{g, \tilde{f}_{j}}^{\lambda^{j}}-c_{j},\left.v^{j}\right|_{A_{j}^{+}\left(c_{j}\right)}\right\rangle+\left\langle W_{g, \tilde{f}_{j}}^{\lambda^{j}}-c_{j},\left.\left(v^{j}-\xi^{j}\right)\right|_{A_{j}^{-}\left(c_{j}\right)}\right\rangle \geqslant 0 .
\end{aligned}
$$

Summing up these inequalities over all $j \in I^{+}$, we conclude from Lemma 3.5 with the (convex) set $\mathfrak{E}=\mathscr{E}_{g, \mathbf{f}^{+}}^{\xi^{+}}\left(\mathbf{A}^{+}, \mathbf{a}^{+} ; D\right)$ that $\lambda$ satisfies $\left(\mathrm{i}^{\prime}\right)$.

9.2. Proof of Theorem 7.4. For any $x \in D$ consider the inverse $K_{x}$ of $\mathrm{Cl}_{\overline{\mathbb{R}^{n}}} A_{p}$ relative to $S(x, 1), \overline{\mathbb{R}^{n}}$ being the one-point compactification of $\mathbb{R}^{n}$. Since $K_{x}$ is compact, there exists the (unique) $\kappa_{\alpha}$-equilibrium measure $\gamma_{x} \in \mathscr{E}_{\alpha}^{+}\left(K_{x} ; \mathbb{R}^{n}\right)$ on $K_{x}$, possessing the properties $\left\|\gamma_{x}\right\|_{\alpha}^{2}=\gamma_{x}\left(K_{x}\right)=c_{\alpha}\left(K_{x}\right)$,

$$
\kappa_{\alpha}\left(\cdot, \gamma_{x}\right)=1 \quad \text { n.e. on } K_{x},
$$

and $\kappa_{\alpha}\left(\cdot, \gamma_{x}\right) \leqslant 1$ on $\mathbb{R}^{n}$. Note that $\gamma_{x} \neq 0$, for $c_{\alpha}\left(K_{x}\right)>0$ in consequence of $c_{\alpha}\left(A_{p}\right)>0$ (see [19, Chapter IV, Section 5, $\left.\left.\mathrm{n}^{\circ} 19\right]\right)$. We assert that, under the 
stated requirements,

$$
S_{\mathbb{R}^{n}}^{\gamma_{x}}= \begin{cases}\breve{K}_{x} & \text { if } \alpha<2, \\ \partial_{\mathbb{R}^{n}} K_{x} & \text { if } \alpha=2 .\end{cases}
$$

The latter equality in (9.12) follows from [19, Chapter II, Section 3, $\mathrm{n}^{\circ} 13$ ]. To establish the former equality, ${ }^{15}$ we first note that $S_{\mathbb{R}^{n}}^{\gamma_{x}} \subset \breve{K}_{x}$ by the $c_{\alpha}$-absolute continuity of $\gamma_{x}$. As for the converse inclusion, assume on the contrary that there is $x_{0} \in \breve{K}_{x}$ such that $x_{0} \notin S_{\mathbb{R}^{n}}^{\gamma_{x}}$. Choose $r>0$ with the property $\bar{B}\left(x_{0}, r\right) \cap S_{\mathbb{R}^{n}}^{\gamma_{x}}=\varnothing$. But $c_{\alpha}\left(B\left(x_{0}, r\right) \cap \breve{K}_{x}\right)>0$, hence by (9.11) there exists $y \in B\left(x_{0}, r\right)$ such that $\kappa_{\alpha}\left(y, \gamma_{x}\right)=1$. The function $\kappa_{\alpha}\left(\cdot, \gamma_{x}\right)$ is $\alpha$-harmonic on $B\left(x_{0}, r\right)$ [19, Chapter I, Section 5, $\left.\mathrm{n}^{\circ} 20\right]$, continuous on $\bar{B}\left(x_{0}, r\right)$, and takes at $y \in B\left(x_{0}, r\right)$ its maximum value 1. Applying [19, Theorem 1.28] we obtain $\kappa_{\alpha}\left(\cdot, \gamma_{x}\right)=1 m_{n}$-a.e. on $\mathbb{R}^{n}$, hence everywhere on $\breve{K}_{x}^{c}$ by the continuity of $\kappa_{\alpha}\left(\cdot, \gamma_{x}\right)$ on $\left(S_{\mathbb{R}^{n}}^{\gamma_{x}}{ }^{c} \supset \breve{K}_{x}^{c}\right.$, and altogether n.e. on $\mathbb{R}^{n}$ by (9.11). This means that $\gamma_{x}$ serves as the $\alpha$-Riesz equilibrium measure on the whole of $\mathbb{R}^{n}$, which is impossible.

Based on (6.3) and the integral representation (4.3), we then arrive at the claimed relation (7.6) in view of the fact that, for every $x \in D, \varepsilon_{x}^{\prime}$ is the Kelvin transform of the equilibrium measure $\gamma_{x}[15$, Section 3.3].

9.3. Proof of Theorem 7.5. Combining (7.5) (with $\zeta=0$ ) and (7.8) yields the first line in (7.9), while the second line is given by (9.2). Substituting the first relation from (7.9) into (7.3) shows that under the stated assumptions the number $c_{1}$ from Theorem 7.3 is $>0$, while (7.2) now takes the (equivalent) form

$$
\kappa_{\alpha}(\cdot, \lambda) \geqslant c_{1}>0 \quad\left(\xi^{1}-\lambda^{+}\right) \text {-a.e. }
$$

Having rewritten (7.3) as

$$
\kappa_{\alpha}\left(\cdot, \lambda^{+}\right) \leqslant \kappa_{\alpha}\left(\cdot, \lambda^{-}\right)+c_{1} \quad \lambda^{+} \text {-a.e., }
$$

we infer from [19, Theorems $1.27,1.29,1.30]$ that the same inequality holds on all of $\mathbb{R}^{n}$, which amounts to (7.11). In turn, (7.11) yields (7.10) when combined with (9.13). It follows directly from Theorem 7.3 that (7.10) and (7.11) together with the relation $\kappa_{\alpha}(\cdot, \lambda)=0$ n.e. on $D^{c}$ determine uniquely the solution $\lambda$ to problem (7.7) among the admissible measures.

Assume now that $\kappa_{\alpha}\left(\cdot, \xi^{1}\right)$ is continuous on $D$. Then so is $\kappa_{\alpha}\left(\cdot, \lambda^{+}\right)$. Indeed, since $\kappa_{\alpha}\left(\cdot, \lambda^{+}\right)$is 1.s.c. and since $\kappa_{\alpha}\left(\cdot, \lambda^{+}\right)=\kappa_{\alpha}\left(\cdot, \xi^{1}\right)-\kappa_{\alpha}\left(\cdot, \xi^{1}-\lambda^{+}\right)$ with $\kappa_{\alpha}\left(\cdot, \xi^{1}\right)$ continuous and $\kappa_{\alpha}\left(\cdot, \xi^{1}-\lambda^{+}\right)$1.s.c., it follows that $\kappa_{\alpha}\left(\cdot, \lambda^{+}\right)$is

\footnotetext{
${ }^{15} \mathrm{We}$ have brought here this proof, since we did not find a reference for this possibly known assertion.
} 
also upper semicontinuous, hence continuous. Therefore, by the continuity of $\kappa_{\alpha}\left(\cdot, \lambda^{+}\right)$on $D,(7.10)$ implies (7.12). Thus, by (7.9) and (7.12),

$$
g\left(\cdot, \lambda^{+}\right)=c_{1} \quad \text { on } S_{D}^{\xi^{1}-\lambda^{+}},
$$

which implies (7.13) in view of [11, Lemma 3.2.2] (with $X=D$ and $\kappa=g$ ).

Omitting now the requirement of continuity of $\kappa_{\alpha}\left(\cdot, \xi^{1}\right)$, assume further that $\alpha<2$ and $m_{n}\left(D^{c}\right)>0$. If on the contrary (7.14) is not fulfilled, then there is $x_{0} \in S_{D}^{\xi^{1}}$ such that $x_{0} \notin S_{D}^{\lambda^{+}}$, and therefore one can choose $r>0$ so that

$$
\bar{B}\left(x_{0}, r\right) \subset D \quad \text { and } \quad \bar{B}\left(x_{0}, r\right) \cap S_{D}^{\lambda^{+}}=\varnothing .
$$

Then $\left(\xi^{1}-\lambda^{+}\right)\left(\bar{B}\left(x_{0}, r\right)\right)>0$, and hence there exists $y \in \bar{B}\left(x_{0}, r\right)$ with the property $\kappa_{\alpha}(y, \lambda)=c_{1}$ (cf. $\left.(7.10)\right)$, or equivalently

$$
\kappa_{\alpha}\left(y, \lambda^{+}\right)=\kappa_{\alpha}\left(y, \lambda^{-}\right)+c_{1} .
$$

Since $\kappa_{\alpha}\left(\cdot, \lambda^{+}\right)$is $\alpha$-harmonic on $B\left(x_{0}, r\right)$ and continuous on $\bar{B}\left(x_{0}, r\right)$ and since $\kappa_{\alpha}\left(\cdot, \lambda^{-}\right)+c_{1}$ is $\alpha$-superharmonic on $\mathbb{R}^{n}$, we conclude from (7.11) and (9.15) with the aid of [19, Theorem 1.28] that

$$
\kappa_{\alpha}(\cdot, \lambda)=\kappa_{\alpha}\left(\cdot, \lambda^{-}\right)+c_{1} \quad m_{n} \text {-a.e. on } \mathbb{R}^{n} .
$$

This implies $c_{1}=0$, because by (4.2) and $(6.3), \kappa_{\alpha}\left(\cdot, \lambda^{+}\right)=\kappa_{\alpha}\left(\cdot,\left(\lambda^{+}\right)^{\prime}\right)=$ $\kappa_{\alpha}\left(\cdot, \lambda^{-}\right)$holds n.e. on $D^{c}$, and hence $m_{n}$-a.e. on $D^{c}$, which is a contradiction.

Similar arguments enable us to establish (7.15). Indeed, if (7.15) were not fulfilled at some $x_{1} \in D \backslash S_{D}^{\lambda^{+}}$, then (9.15) would hold with $x_{1}$ in place of $y$ (cf. (7.11)) and, furthermore, one could choose $r>0$ so that (9.14) would be valid with $x_{1}$ in place of $x_{0}$. Therefore, since $\kappa_{\alpha}\left(\cdot, \lambda^{+}\right)$is $\alpha$-harmonic on $B\left(x_{1}, r\right)$ and continuous on $\bar{B}\left(x_{1}, r\right)$ and since $\kappa_{\alpha}\left(\cdot, \lambda^{-}\right)+c_{1}$ is $\alpha$-superharmonic on $\mathbb{R}^{n}$, we would arrive again at (9.16) and hence at $c_{1}=0$, which is impossible.

\section{Examples}

The purpose of the examples below is to illustrate the assertions from Section 7.1. Note that in either Example 10.1 or Example 10.2 the set $A_{2}=D^{c}$ is not $\alpha$-thin at infinity.

ExAmple 10.1. Let $n \geqslant 3, \alpha<2, D=B_{r}:=B(0, r)$, where $r \in(0, \infty)$, and let $I^{+}=\{1\}, A_{1}=D, \mathbf{a}=\mathbf{1}, \mathbf{f}=\mathbf{0}$. Define $\xi^{1}:=q \lambda_{r}$, where $q \in(1, \infty)$ and $\lambda_{r}$ is the $\kappa_{\alpha}$-capacitary measure on $\bar{B}_{r}:=\bar{B}(0, r)$ (Remark 2.3). As follows from [19, Chapter II, Section 3, $\left.\mathrm{n}^{\circ} 13\right], \xi^{1} \in \mathscr{E}_{\alpha}^{+}\left(A_{1}, q ; \mathbb{R}^{n}\right), S_{D}^{\xi^{1}}=D$ and $\kappa_{\alpha}\left(\cdot, \xi^{1}\right)$ is continuous on $\mathbb{R}^{n}$. Since $\mathbf{f}=\mathbf{0}$, Problem 3.2 reduces to problem (7.7) of minimizing $\kappa_{\alpha}(\mu, \mu)$ over all $\mu \in \mathscr{E}_{\alpha}\left(\mathbb{R}^{n}\right)$ such that $\mu^{+} \in \mathscr{E}_{\alpha}^{\xi^{1}}\left(A_{1}, 1 ; \mathbb{R}^{n}\right)$ and $\mu^{-} \in$ 
$\mathscr{E}_{\alpha}^{+}\left(A_{2}, 1 ; \mathbb{R}^{n}\right)$, which by Theorem 6.1 is equivalent to the problem of minimizing $g_{D}^{\alpha}(v, v)$ over $\mathscr{E}_{g_{D}^{\alpha}}^{\xi^{1}}\left(A_{1}, 1 ; D\right)$. According to Theorems 7.1, 6.1 and Corollary 3.1, these two constrained minimum energy problems are uniquely solvable (no short-circuit occurs between $D$ and $D^{c}$ ), and their solutions, denoted respectively by $\lambda_{\alpha, \mathbf{A}}=\lambda^{+}-\lambda^{-}$and $\lambda_{g, A_{1}}$, are related to each other as follows:

$$
\lambda_{\alpha, \mathbf{A}}=\lambda_{g, A_{1}}-\lambda_{g, A_{1}}^{\prime}
$$

Furthermore, by (7.6), (7.13) and (7.14),

$$
\begin{gathered}
S_{D}^{\lambda^{+}}=S_{D}^{\lambda_{g, A_{1}}}=S_{D}^{\xi^{1}}=D, \quad S_{\mathbb{R}^{n}}^{\lambda^{-}}=D^{c}, \\
c_{g_{D}^{\alpha}}\left(S_{D}^{\xi^{1}-\lambda^{+}}\right)<\infty,
\end{gathered}
$$

while by (7.9), (7.11) and (7.12),

$$
\begin{gathered}
\kappa_{\alpha}\left(\cdot, \lambda_{\alpha, \mathbf{A}}\right)= \begin{cases}c_{1} & \text { on } S_{D}^{\xi^{1}-\lambda^{+}}, \\
0 & \text { on } D^{c},\end{cases} \\
\kappa_{\alpha}\left(\cdot, \lambda_{\alpha, \mathbf{A}}\right) \leqslant c_{1} \quad \text { on } D \backslash S_{D}^{\xi^{1}-\lambda^{+}},
\end{gathered}
$$

where $c_{1}>0$. (In (10.2) we have used the fact that for the given $\alpha$ and $D$, $I_{\alpha, D^{c}}=\varnothing$.) Moreover, according to Theorem 7.3, relations (10.2) and (10.3) determine uniquely the solution $\lambda_{\alpha, \mathbf{A}}$ among the admissible measures.

Example 10.2. Let $n=3, \quad \alpha=2, \quad \mathbf{f}=\mathbf{0}, \quad \mathbf{a}=1$. Define $D:=\{x=$ $\left.\left(x_{1}, x_{2}, x_{3}\right) \in \mathbb{R}^{3}: x_{1}>0\right\}$ and $A_{1}:=\sum_{k \in \mathbb{N}} K_{k}$, where

$$
K_{k}:=\left\{\left(x_{1}, x_{2}, x_{3}\right) \in D: x_{1}=k^{-1}, x_{2}^{2}+x_{3}^{2} \leqslant k^{2}\right\}, \quad k \in \mathbb{N} .
$$

Let $\lambda_{k}$ be the $\kappa_{2}$-capacitary measure on $K_{k}$ (Remark 2.3 ); hence $\lambda_{k}\left(K_{k}\right)=1$ and $\left\|\lambda_{k}\right\|_{2}^{2}=\pi^{2} /(2 k)$ by [19, Chapter II, Section 3, $\left.\mathrm{n}^{\circ} 14\right]$. Define

$$
\xi^{1}:=\sum_{k \in \mathbb{N}} k^{-2} \lambda_{k}
$$

In the same manner as in the proof of Theorem 7.2 one can see that $\xi^{1}$ is a positive Radon measure carried by $A_{1}$ with $\kappa_{2}\left(\xi^{1}, \xi^{1}\right)<\infty$ and $\xi^{1}\left(A_{1}\right) \in(1, \infty)$. By Theorem 7.1, Problem 3.2 for the constraint $\xi=\left(\xi^{1}, \infty\right)$ and the condenser $\mathbf{A}=\left(A_{1}, D^{c}\right)$ has therefore a (unique) solution $\lambda_{\alpha, \mathbf{A}}=\lambda^{+}-\lambda^{-}$(no short-circuit occurs between $A_{1}$ and $D^{c}$ ), although $D^{c} \cap \mathrm{Cl}_{\mathbb{R}^{3}} A_{1}=\partial D$ and hence

$$
c_{2}\left(D^{c} \cap \mathrm{Cl}_{\mathbb{R}^{3}} A_{1}\right)=\infty .
$$

Furthermore, since each $\kappa_{2}\left(\cdot, \lambda_{k}\right), k \in \mathbb{N}$, is continuous on $\mathbb{R}^{n}$ and bounded from above by $\pi^{2} /(2 k)$, the potential $\kappa_{2}\left(\cdot, \xi^{1}\right)$ is continuous on $\mathbb{R}^{n}$ by the 
uniform convergence of the sequence $\sum_{k \in \mathbb{N}} k^{-2} \kappa_{2}\left(\cdot, \lambda_{k}\right)$. Hence (10.1), (10.2) and (10.3) also hold in the present case with $\alpha=2$, again with $c_{1}>0$, and relations (10.2) and (10.3) determine uniquely the solution $\lambda_{\alpha, \mathbf{A}}$ among the admissible measures. Also note that $S_{\mathbb{R}^{n}}^{\lambda^{-}}=\partial D$ according to (7.6).

\section{Acknowledgement}

The authors express their sincere gratitude to the anonymous Referee for valuable suggestions, helping us in improving the exposition of the paper.

\section{References}

[1] J. Bliedtner and W. Hansen, Potential Theory: An Analytic and Probabilistic Approach to Balayage, Springer, Berlin, 1986.

[2 ] N. Bourbaki, Elements of Mathematics. General Topology. Chapters 1-4, Springer, Berlin, 1989.

[3] N. Bourbaki, Elements of Mathematics. Integration. Chapters 1-6, Springer, Berlin, 2004.

[4] M. Brelot, On Topologies and Boundaries in Potential Theory, Lecture Notes in Math. 175, Springer, Berlin, 1971.

[5] H. Cartan, Théorie du potentiel newtonien: énergie, capacité, suites de potentiels, Bull. Soc. Math. Fr. 73 (1945), 74-106.

[6] H. Cartan and J. Deny, Le principe du maximum en théorie du potentiel et la notion de fonction surharmonique, Acta Sci. Math. (Szeged) 12 (1950), 81-100.

[7] J. Deny, Les potentiels d'énergie finie, Acta Math. 82 (1950), 107-183.

[8] J. Deny, Sur la définition de l'énergie en théorie du potentiel, Ann. Inst. Fourier (Grenoble) 2 (1950), 83-99.

[ 9 ] P. D. Dragnev, B. Fuglede, D. P. Hardin, E. B. Saff and N. Zorii, Minimum Riesz energy problems for a condenser with touching plates, Potential Anal. 44 (2016), 543-577.

[10] R. Edwards, Functional Analysis. Theory and Applications, Holt, Rinehart and Winston, New York, 1965.

[11] B. Fuglede, On the theory of potentials in locally compact spaces, Acta Math. 103 (1960), $139-215$.

[12] B. Fuglede, Capacity as a sublinear functional generalizing an integral, Mat.-Fys. Medd. Danske Vid. Selsk. 38 (1971), no. 7, 44 pp.

[13] B. Fuglede, Symmetric function kernels and sweeping of measures, Anal. Math. 42 (2016), 225-259.

[14] B. Fuglede and N. Zorii, Constrained Gauss variational problems for a condenser with intersecting plates, Ann. Polon. Math. 120 (2017), 227-260.

[15] B. Fuglede and N. Zorii, Green kernels associated with Riesz kernels, Ann. Acad. Sci. Fenn. Math. 43 (2018), 121-145.

[16] B. Fuglede and N. Zorii, An alternative concept of Riesz energy of measures with application to generalized condensers, Potential Anal. (2018). https://doi.org/10.1007/s11118-0189709-3

[17] C. F. Gauss, Allgemeine Lehrsätze in Beziehung auf die im verkehrten Verhältnisse des Quadrats der Entfernung wirkenden Anziehungs- und Abstoßungs-Kräfte (1839), Werke 5 (1867), 197-244. 
[18] H. Harbrecht, W. L. Wendland and N. Zorii, Riesz minimal energy problems on $C^{k-1, k} k_{-}$ manifolds, Math. Nachr. 287 (2014), 48-69.

[19] N. S. Landkof, Foundations of Modern Potential Theory, Springer, Berlin, 1972.

[20] G. Of, W. L. Wendland and N. Zorii, On the numerical solution of minimal energy problems, Complex Var. Elliptic Equ. 55 (2010), 991-1012.

[21] M. Ohtsuka, On potentials in locally compact spaces, J. Sci. Hiroshima Univ. Ser. A-1, 25 (1961), 135-352.

[22] N. Zorii, Interior capacities of condensers with countably many plates in locally compact spaces, Hiroshima Math. J. 40 (2010), 273-313.

[23] N. Zorii, Interior capacities of condensers in locally compact spaces, Potential Anal. 35 (2011), 103-143.

[24] N. Zorii, Constrained energy problems with external fields for vector measures, Math. Nachr. 285 (2012), 1144-1165.

[25] N. Zorii, Equilibrium problems for infinite dimensional vector potentials with external fields, Potential Anal. 38 (2013), 397-432.

[26] N. Zorii, Necessary and sufficient conditions for the solvability of the Gauss variational problem for infinite dimensional vector measures, Potential Anal. 41 (2014), 81-115.

\author{
Bent Fuglede \\ Department of Mathematical Sciences \\ University of Copenhagen \\ 2100 Copenhagen, Denmark \\ E-mail: fuglede@math.ku.dk \\ Natalia Zorii \\ Institute of Mathematics \\ National Academy of Sciences of Ukraine \\ Tereshchenkivska 3, 01601, Kyiv-4, Ukraine \\ E-mail: natalia.zorii@gmail.com
}

